\title{
Dynamic expression of homeostatic ion channels in differentiated cortical astrocytes in vitro
}

\author{
Francesco Formaggio ${ }^{1}$ Martina Fazzina ${ }^{1,2} \cdot$ Raúl Estévez $^{3,4} \cdot$ Marco Caprini $^{1} \cdot$ Stefano Ferroni ${ }^{1}$ (1)
}

Received: 9 June 2021 / Revised: 2 September 2021 / Accepted: 24 September 2021 / Published online: 4 November 2021

(c) The Author(s) 2021, corrected publication 2022

\begin{abstract}
The capacity of astrocytes to adapt their biochemical and functional features upon physiological and pathological stimuli is a fundamental property at the basis of their ability to regulate the homeostasis of the central nervous system (CNS). It is well known that in primary cultured astrocytes, the expression of plasma membrane ion channels and transporters involved in homeostatic tasks does not closely reflect the pattern observed in vivo. The individuation of culture conditions that promote the expression of the ion channel array found in vivo is crucial when aiming at investigating the mechanisms underlying their dynamics upon various physiological and pathological stimuli. A chemically defined medium containing growth factors and hormones (G5) was previously shown to induce the growth, differentiation, and maturation of primary cultured astrocytes. Here we report that under these culture conditions, rat cortical astrocytes undergo robust morphological changes acquiring a multi-branched phenotype, which develops gradually during the 2-week period of culturing. The shape changes were paralleled by variations in passive membrane properties and background conductance owing to the differential temporal development of inwardly rectifying chloride $\left(\mathrm{Cl}^{-}\right)$and potassium $\left(\mathrm{K}^{+}\right)$currents. Confocal and immunoblot analyses showed that morphologically differentiated astrocytes displayed a large increase in the expression of the inward rectifier $\mathrm{Cl}^{-}$and $\mathrm{K}^{+}$ channels ClC-2 and Kir4.1, respectively, which are relevant ion channels in vivo. Finally, they exhibited a large diminution of the intermediate filaments glial fibrillary acidic protein (GFAP) and vimentin which are upregulated in reactive astrocytes in vivo. Taken together the data indicate that long-term culturing of cortical astrocytes in this chemical-defined medium promotes a quiescent functional phenotype. This culture model could aid to address the regulation of ion channel expression involved in CNS homeostasis in response to physiological and pathological challenges.
\end{abstract}

Keywords Cultured astrocytes $\cdot$ Ion channels $\cdot \mathrm{K}^{+}$channel $\cdot \mathrm{Cl}^{-}$channel $\cdot$ Brain homeostasis

\section{Introduction}

In the last decades, a large body of evidence have accumulated showing that in the central nervous system (CNS), astroglial cells are actively involved in the maintenance of

Stefano Ferroni

stefano.ferroni@unibo.it

1 Department of Pharmacy and Biotechnology, University of Bologna, Via San Donato 19/2, 40127 Bologna, Italy

2 Present Address: Department for Life Quality Studies, University of Bologna, Rimini, Italy

3 Departament de Ciències Fisiològiques, IDIBELL-Institute of Neurosciences, Universitat de Barcelona, Barcelona, Spain

4 Centro de Investigación Biomédica en Red Sobre Enfermedades Raras (CIBERER), Instituto de Salud Carlos III, Madrid, Spain neuronal health through various mechanisms including neuronal metabolic and trophic supports, the homeostatic regulation of the perineuronal milieu, and neurovascular support [82]. The ability of astrocytes to influence the physiological response of neuronal circuits is also corroborated by the wealth of information reporting that, as consequence of a variety of acute and chronic pathological conditions, astrocytes can become activated (reactive) and respond to various insults through alterations of morphological, molecular, and biochemical processes [91].

Fully functional astrocytes in vivo are endowed with a variety of ion channels and transporters some of which have unequivocally been shown to have housekeeping homeostatic roles [61, 83]. Astrocytes express a large background potassium $\left(\mathrm{K}^{+}\right)$conductance carried mainly by the weakly inward-rectifier $\mathrm{K}^{+}$channel 4.1 (Kir4.1); this $\mathrm{K}^{+}$conductance sets the very negative membrane 
potential, contributes to the maintenance of the extracellular $\mathrm{K}^{+}$homeostasis, and regulates the efficacy of glutamate uptake $[6,15,55,56]$. Kir4.1 is strongly developmentally regulated being virtually absent at birth and showing a sharp increment in expression at 10 days postnatal [75]. Furthermore, Kir4.1 channel is also downregulated under various pathological conditions [5, 26, 44, 58, 67]. Of note, when studied in culture conditions, Kir channels are not functionally expressed $[1,3,21]$. Hence the identification of culture conditions that can more closely reflect the in vivo situation is mandatory to address the molecular mechanisms that contribute to the development and maintenance of a mature functional phenotype and those that induce reactive astrogliosis in which the expression of ion channels is altered.

We previously reported that a Kir conductance and an inward rectifier chloride $\left(\mathrm{Cl}^{-}\right)$current were observed when cultured astrocytes were exposed for long term to conditions that elevate cytosolic cyclic AMP, which induces their morphological differentiation from flat polygonal to a phenotype with long thick processes [21]. Other studies reported that cultured rodent astrocytes, grown in the presence of a morphologically differentiating supplement (G5) containing growth factors and hormones [50], displayed the upregulation of glutamate uptake through mechanisms mediated, at least partially, by augmented levels of the sodium-dependent glutamate transporters GLT-1 and GLAST [7, 27, 85]. There is also indication that G5 augmented the saxitoxin-sensitive voltage-gated sodium $\left(\mathrm{Na}^{+}\right)$channels [90]. At variance, cultured astrocytes from rat corpus callosum exposed to G5 did not evidence any significant alterations in $\mathrm{K}^{+}$conductance [72].

Because of these variable effects of G5 supplementation on the expression of plasma membrane homeostatic proteins in this work, we sought to determine whether the morphological differentiation of primary astrocytes induced by adding G5 to the culture medium devoid of fetal bovine serum (FBS) was associated to the expression of homeostatic ion channels which are not present in conventionally cultured astrocytes. Our results show that rat cortical astrocytes cultured for long term in G5-containing medium acquired a differentiated morphological phenotype and displayed $\mathrm{K}^{+}$and $\mathrm{Cl}^{-}$conductance which are found in mature astrocytes. Functional and molecular analyses indicate that G5-treated astrocytes exhibited an increase in Kir4.1 and ClC-2 proteins. This culture protocol could be suitable to address the dynamic mechanisms that influence the expression of functionally relevant ion channels in long-term cultured astrocytes under physiological and pathological conditions.

\section{Materials and methods}

\section{Preparation of primary cultures of neonatal rat cortical astrocytes}

All the experiments were performed according to the Italian law on protection of laboratory animals, with the approval of bioethical committees of the University of Bologna (AEDB0.2) and of the Ministry of Health (protocol number 83/2017-PR) and under the supervision of the veterinary commission for animal care and comfort at the University of Bologna.

Primary cultures of cortical astrocytes from newborn (1-2 days) Sprague-Dawley breeding pairs (Charles River, Italy) were obtained according to the standard method [47] with some modifications [21] to obtain highly purified cultures composed of more than $95 \%$ astrocytes. Cultured flasks were maintained in DMEM containing GlutaMAX ${ }^{\mathrm{TM}}-\mathrm{I}$ and $4.5 \mathrm{~g} / \mathrm{L}$ D-glucose, with $10 \%$ of heat-inactivated fetal bovine serum (FBS) and penicillin-streptomycin $(100 \mathrm{U} / \mathrm{mL}$ and $100 \mu \mathrm{g} / \mathrm{mL}$ respectively). All products were from Gibco-BRL.

\section{G5 treatment of primary cortical astrocytes}

Confluent astroglial monolayers were enzymatically detached using trypsin (Gibco-BRL) and plated onto Petri dishes at low density (see below). Two days after, FBS was reduced to $3 \%$, and G5 supplement (Sigma-Aldrich) was added $(10 \mu \mathrm{L} /$ $\mathrm{mL}$ ) to the culture medium. G5 is a chemical-defined supplement based on a formulation composed of growth factors, hormones, and micronutrients that induces the morphological differentiation of cultured astrocytes and lowers proliferation rate [50]. After 2 days of culturing in medium containing 3\% FBS and G5, at the next medium change FBS was removed and G5 was present for the remaining period of culturing up to 16 days (days of treatment, DOT). G5 was freshly added every 4-5 DOT when changing the medium (Table 1). Untreated astrocytes were maintained in the same culture conditions in the absence of G5.

\section{Electrophysiology}

For electrophysiological experiments, untreated astrocytes were plated in 35-mm Petri dishes at a density of 24,000 cells per dish, whereas, to avoid cell-cell contact, highly branched G5-treated astrocytes were plated at 8,000 or 12,000 cells per dish. Whole-cell patch-clamp experiments were carried out as previously described [20,21]. During each day of experiment, recordings were performed alternating measurements in G5-treated and untreated (ctrl) astrocytes. Petri dishes were 
mounted on the stage of an inverted microscope equipped with phase-contrast optics (Nikon Diaphot). Patch-clamp pipettes were prepared from borosilicate glass capillaries to have a tip resistance of 2-4 M $\Omega$ when filled with the standard intracellular solution. Membrane currents were amplified with an EPC-7 amplifier (List Electronic, Darmstadt Germany) and low-pass filtered at $3 \mathrm{kHz}$ before acquisition. Stimulation and analysis were performed with pClamp 6 software (Axon Instrument, Foster City, CA, USA) and Origin 6.0 (MicroCal, Northampton, MA, USA). All experiments were carried out at room temperature $\left(20-24^{\circ} \mathrm{C}\right)$. Series resistance values were below $10-12 \mathrm{M} \Omega$ and were corrected for by $40-60 \%$ with the analog circuit of the amplifier. Cell capacitance of the recorded cell was obtained by the amplifier reading of the capacitive transient cancelation.

The standard external (bath) solution was (mM) 140 $\mathrm{NaCl}, 4 \mathrm{KCl}, 2 \mathrm{MgCl}_{2}, 2 \mathrm{CaCl}_{2}, 10$ HEPES 5 glucose, $\mathrm{pH}$ 7.4 adjusted with $\mathrm{NaOH}$, and osmolarity $310 \mathrm{mOsm}$ corrected with mannitol. The standard intracellular (pipette) solution was composed of (mM) $144 \mathrm{KCl}, 2 \mathrm{MgCl}_{2}, 5$ EGTA, 10 HEPES, pH 7.2 adjusted $\mathrm{KOH}$, and osmolarity 290 mOsm with mannitol. When using external solution with different ionic compositions, salts were replaced equimolarly. To isolate $\mathrm{Cl}^{-}$currents, the external solution used was (mM) $120 \mathrm{CsCl}, 2 \mathrm{MgCl}_{2}, 2 \mathrm{CaCl}_{2}, 102$-[Tris (hydroxymethyl)-methylamino]-ethanesulfonic acid (TES), 5 glucose, pH 7.2 adjusted with $\mathrm{CsOH}$, and osmolarity $\sim 310$ mOsm with mannitol. The pipette solution was composed of (mM) $125 \mathrm{CsCl}, 2 \mathrm{MgCl}_{2}, 5$ EGTA, $10 \mathrm{TES}, \mathrm{pH}$ 7.2 adjusted with $\mathrm{CsOH}$, and osmolarity $\sim 290 \mathrm{mOsm}$ with mannitol. Changes of solutions around the recorded cells were performed by using a gravity-driven, local perfusion system at a flow rate of $\sim 200 \mu \mathrm{L} / \mathrm{min}$ and positioned within $\sim 100 \mu \mathrm{m}$ of the recorded cell.

\section{Western blot}

The primary and secondary antibodies used are listed in Table 2. Astrocytes were seeded in 60-mm Petri dishes, and cell lysates from G5-treated and untreated astrocytes were obtained at 5-7 and 12-16 DOT. Cells were lysed with 200 $\mu \mathrm{L}$ of RIPA buffer and quantified with the Bradford method, as previously described [24]. Proteins were separated in a 10-12\% SDS-polyacrylamide, blotted into PVDF membrane, and incubated $1 \mathrm{~h}$ at room temperature (RT) in blocking solution made of PBST $0.1 \%$ containing 5\% BSA (SigmaAldrich). Membranes were incubated with primary antibodies overnight at $4{ }^{\circ} \mathrm{C}$ in PBST $0.1 \%$ containing $1 \%$ BSA. The following day, they were probed with IgG horseradish

Table 1 Timeline of G5 treatment and data collection

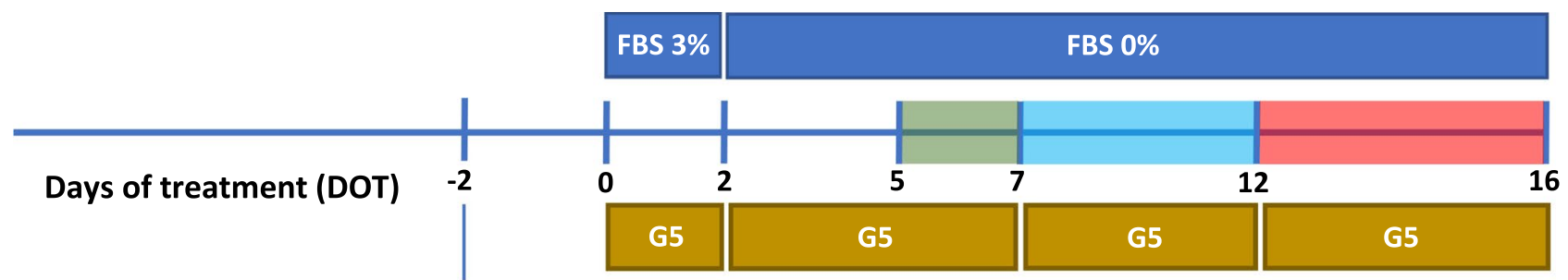

Petri dish seeding

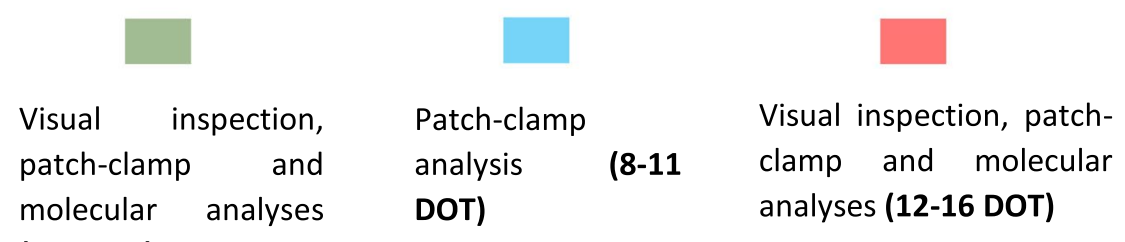

(5-7 DOT)

Table 2 List of antibodies used for western blot analysis

\begin{tabular}{ll}
\hline Primary antibodies & Mouse anti-Kir4.1 (Santa-Cruz Biotechnology, sc-293252) (1:500) \\
& Mouse anti-GFAP (Santa-Cruz Biotechnology, sc-33673) (1:1000) \\
& Rabbit anti- $\beta$-actin (Sigma-Aldrich, A2066) (1:1000) \\
& Rabbit anti-ClC-2 (custom-made provided by R. Estévez) (1:500) \\
& Mouse anti-vimentin (Santa-Cruz Biotechnology, sc-6260) (1: 1500) \\
& Mouse anti-GLT-1 (Santa-Cruz Biotechnology, sc-365634) (1:500) \\
& Goat anti-mouse-HRP (Sigma-Aldrich, A4416) (1:5000 for all primary anti- \\
bodies) & Goat anti-rabbit-HRP (Sigma-Aldrich, A12-348) (1:5000 for anti-ClC-2, \\
1:10000 for anti- $\beta$-actin)
\end{tabular}


Table 3 List of antibodies used for immunofluorescence analysis
Primary antibodies
- Rabbit anti-Kir4.1 (Alomone, APC-035) (1:200)
- Rabbit anti-ClC-2 (provided by Prof. R. Estévez) (1:200)
- Chicken anti-GFAP (BioLegend, Poly28294) (1:500)
Secondary antibodies
- Cy-3 donkey anti-chicken (Jackson ImmunoResearch, 703-165-155) (1:400)

peroxidase-conjugated secondary antibodies and developed with the enhancing chemiluminescence detection system (Santa Cruz Biotechnology or Cyanagen-Westar $\eta \mathrm{C}$ ultra 2.0). Blots were visualized with the ChemiDoc ${ }^{\mathrm{TM}}$ (Bio-Rad) imaging system, and densitometric analysis was performed using the Image Lab 6.0 software. Immunoreactive bands were normalized against $\beta$-actin levels.

\section{Immunofluorescence}

The primary and secondary antibodies used are listed in Table 3. For immunofluorescence experiments, cortical astrocytes were plated on glass coverslips coated with polyD-lysine (Sigma-Aldrich) $(100 \mu \mathrm{g} / \mathrm{mL})$ at a concentration of 5000 cells/coverslip for both untreated and G5-treated astrocytes. Immunocytochemical analyses were performed as previously described [24]. Briefly, primary cultured astrocytes were washed twice in PBS, fixed with $4 \%$ paraformaldehyde (Sigma-Aldrich) at RT for $10 \mathrm{~min}$, and washed again twice in PBS for 10 min. To block non-specific staining, astrocytes were incubated for $1 \mathrm{~h}$ at RT in PBS containing 5\% BSA and 0.05\% Triton X-100 (Sigma-Aldrich). After blocking, specimens were probed overnight at $4{ }^{\circ} \mathrm{C}$ with primary antibodies, which were diluted in PBS containing 1\% BSA and $0.05 \%$ Triton X-100. Coverslips were washed 3 times for $20 \mathrm{~min}$ in PBS at RT and incubated $2 \mathrm{~h}$ with secondary antibodies diluted in PBS containing 1\% BSA and 0.05\% Triton X-100. Coverslips were finally washed twice for $5 \mathrm{~min}$ and mounted onto poly-lysine-coated slides (Menzel-Gläser Superfrost, Thermo Scientific) with Fluoromount-G mounting medium (Sigma-Aldrich). Single-plane confocal immunofluorescence images were acquired with an inverted laser scanning confocal microscope (Nikon D-Eclipse C1). To assess staining specificity, astrocytes were also processed in the absence of primary antibodies. All secondary antibodies were conjugated with Cyanine 2/3.

\section{Statistical analysis}

Data are presented as mean \pm standard error or box plots. The D'Agostino-Pearson omnibus test was used to detect consistency of normal distribution. The Grubbs's test was used to detect outliers at the $95 \%$ confidence level. The significance was determined with the Student's $t$-test (paired and unpaired, two-tailed), one-way ANOVA, or Kruskal-Wallis test as appropriate by using GraphPad Prism software (GraphPad Software, Inc., San Diego, CA, USA). A $p$-value $<0.05$ was considered statistically significant.

\section{Results}

\section{Time-dependent morphological differentiation of G5-treated primary cortical astrocytes}

Previous works showed that few days of exposure of cultured astrocytes to growth medium supplemented with G5 resulted in shape changes characterized by a shift from an epithelioid to a process-bearing morphology and an increase in glutamate uptake $[86,87]$. Since we were interested to individuate other functional changes which paralleled the G5 effect on glutamate dynamics, cultured cortical astrocytes were challenged with G5 for a period of up to 16 DOT. Initial analysis was devoted to determining the long-term effect of G5 on the morphology of astrocytes cultured in the absence of FBS. The results show that after 5-7 and 12-16 days in FBS-free control medium (ctrl), astrocytes replated at low density exhibited the epithelioid shape typical of undifferentiated primary cultured rat cortical astrocytes [21] (Fig. 1A, C). Cultured astrocytes exposed to G5 for 5-7 DOT displayed a cell shape with few polarized processes elongating from an irregular cell body (Fig. 1B) and acquired a multi-branched phenotype radially departing from a retracted cell body at 12-16 DOT with G5 (Fig. 1D), These results indicate that the growth of cultured cortical astrocytes in a G5-containing chemical-defined medium promotes shape changes toward a process-bearing phenotype that develops gradually during the 16 DOT.

\section{Electrical membrane properties of G5-treated primary cortical astrocytes}

We next addressed whether the dynamic changes in cell shape observed under these culture conditions were paralleled by alterations in electrical membrane properties. Experiments were carried out with the patch-clamp technique using standard intra- and extracellular solutions. We initially compared the resting membrane potential (RMP) of cortical astrocytes cultured in the absence (ctrl) and presence of G5 measured at various time of G5 treatment. Whereas RMP values were normally distributed in untreated astrocytes at all DOT windows, 
Fig. 1 Time-dependent changes of morphological phenotype in G5-treated primary cultured rat cortical astrocytes. Phase-contrast micrographs of low-density replated astrocytes in FBS-free culture conditions in the absence (ctrl) and presence of G5 supplement for various days of treatment (DOT). A Untreated astrocytes at 5-7 DOT display the typical epithelioid shape. B Subconfluent astrocytes exposed to G5 for 5-7 DOT show few polarized processes elongating from an irregular cell body. $\mathbf{C}$ Untreated subconfluent astrocytes at 12-16 DOT have a flat, epithelioid morphology. D Lowdensity G5-treated astrocytes at 12-16 DOT develop multibranched processes radially departing from retracted cell bodies. Scale bar: $20 \mu \mathrm{m}$
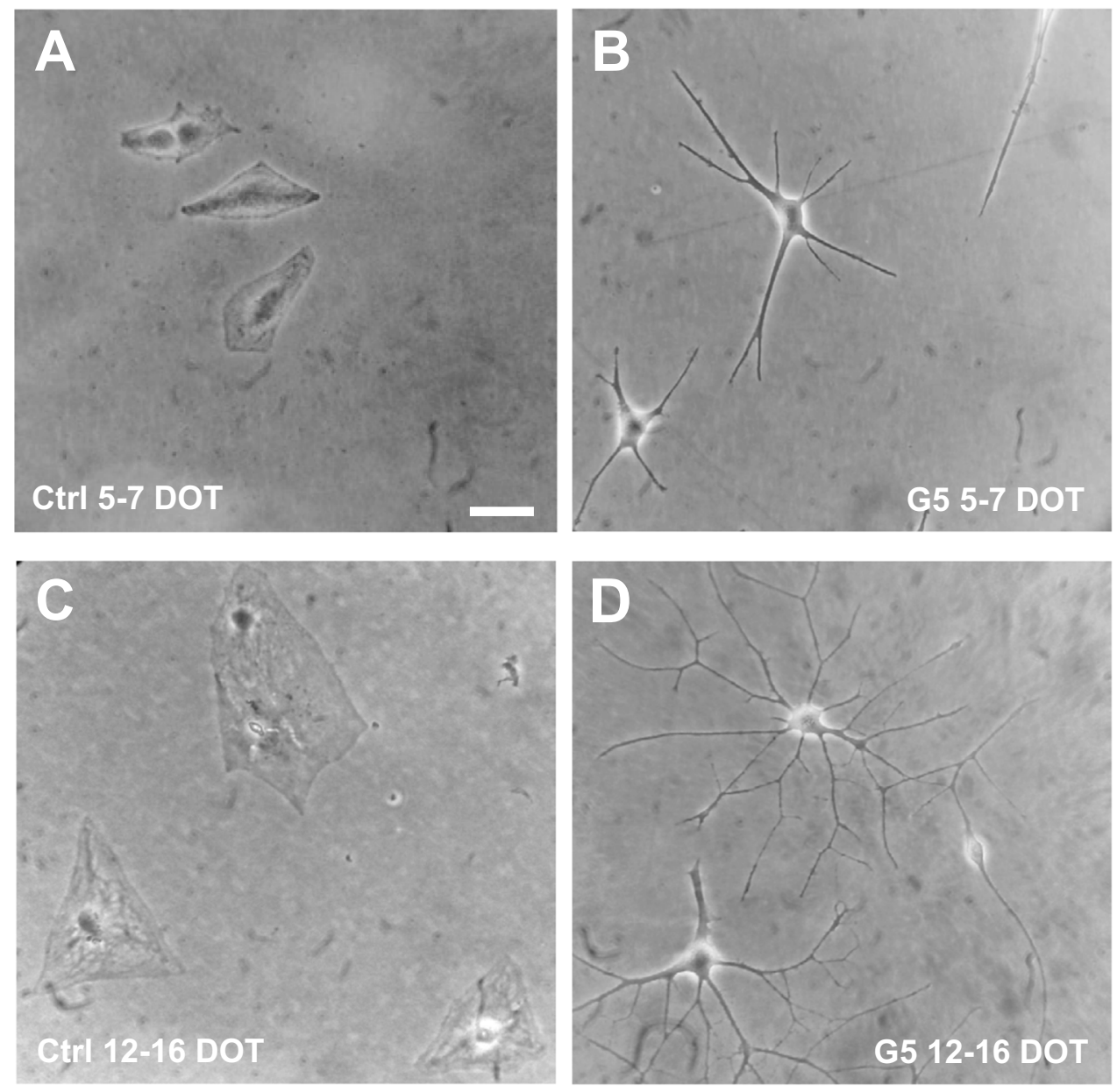

A

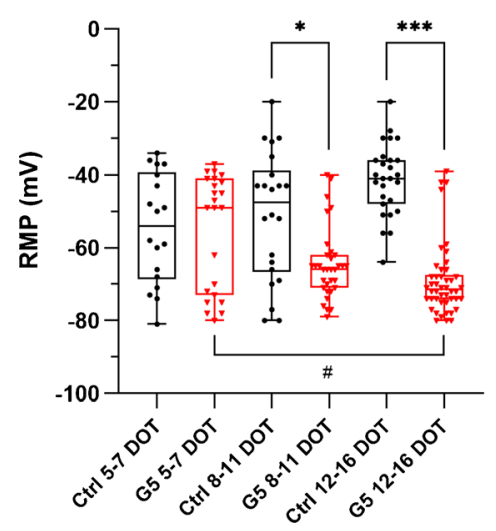

B
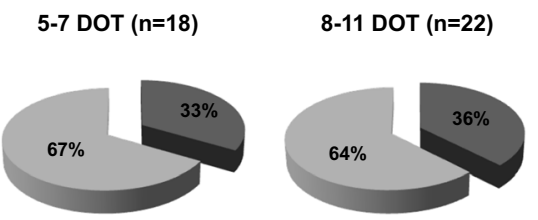

CTRL

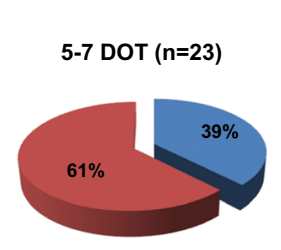

8-11 DOT (n=33)

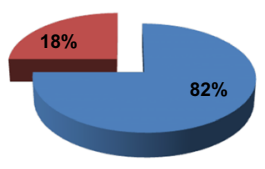

12-16 DOT $(n=27)$

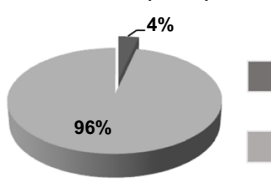

RMP $<-60 \mathrm{mV}$

$\mathrm{RMP}>-60 \mathrm{mV}$

12-16 DOT $(n=46)$

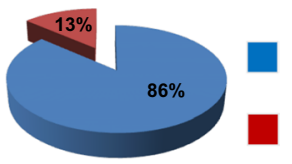

RMP $<-60 \mathrm{mV}$

$\mathrm{RMP}>-60 \mathrm{mV}$
Fig. 2 Time-dependent variations in resting membrane potentials in G5-treated primary cortical astrocytes. A Box plots of resting membrane potential (RMP) values depicting the median and the interquartile range in untreated (ctrl, black) and G5-treated (red) astrocytes at different DOT windows. Untreated astrocytes display a median RMP that remains stable at $\sim-50 \mathrm{mV}$ throughout the whole period of subculturing (5-7 DOT, $n=18 ; 8-11$ DOT, $n=22 ; 12-16$ DOT, $n=27$ ). In G5-treated astrocytes, the median RMP is significantly more hyperpolarized after 8-11 DOT compared to untreated astrocytes
(5-7 DOT, $n=23 ; 8-11$ DOT, $n=32 ; 12-16$ DOT, $n=51) . * p<0.05$, $* * * p<0.001$, and $\# p<0.05$ with Kruskal-Wallis test. Where not indicated, the group differences were non-significant. B Pie graphs of the percentage of astrocytes with RMPs more negative $(<)$ and more positive $(>)$ of $-60 \mathrm{mV}$ in untreated (ctrl) and G5-treated cells. The value of $-60 \mathrm{mV}$ depicts the value of RMP separating the two populations of astrocytes described by the binomial distribution of RMPs in G5-treated astrocytes (Fig. S1). $N$ is the number of cells analyzed in each condition at different DOT windows 
those of G5-treated astrocytes followed a bimodal distribution (Fig. S1). The non-parametric analysis (Fig. 2A and Table 4 of S1) shows that in untreated astrocytes (ctrl), RMPs were not significantly different at all DOT windows. RMP values in untreated and G5-treated astrocytes were not different at 5-7 DOT but were significantly more hyperpolarized in 8-11 and 12-16 DOT G5-treated astrocytes. Moreover, RMPs become more negative with prolonged time of G5 exposure. Because the hyperpolarized RMPs of G5-treated astrocytes could be due to the presence of astrocyte subpopulations [39, 48, 93] with different sensitivity to G5 challenge, we next performed a stratified analysis to assess whether the time of exposure to G5 affected the percentage of cells with RMP higher and lower than a threshold value of $-60 \mathrm{mV}$. This value was chosen because in the bimodal distribution it separated the two RMP populations in G5 astrocytes. The results indicate that whereas in untreated astrocytes the number of cells displaying RMPs more negative than $-60 \mathrm{mV}$ was diminished at $12-16$ DOT (Fig. 2B, upper), in G5-treated cells, there was a positive correlation between time of exposure to G5 and percentage of astrocytes with RMPs more negative than $-60 \mathrm{mV}$ (Fig. 2B, lower).

We next examined whether the gradual hyperpolarizing shift in RMP observed in the G5-treated, morphologically differentiated astrocytes was associated to changes in the other passive membrane properties. The values of cell membrane capacitance $(\mathrm{Cm})$ of untreated astrocytes at all DOT windows were not significantly different. Compared to untreated astrocytes, those cultured in the presence of G5 revealed an increase in $\mathrm{Cm}$ at all DOT (Fig. 3A). We next determined the membrane resistance associated to the background membrane conductance. To unravel the background conductance, astrocytes were voltage clamped at the holding potential $\left(V_{h}\right)$ of $-60 \mathrm{mV}$, and families of 400-ms-long voltage steps of $20 \mathrm{mV}$ increments were delivered from -120 to $+60 \mathrm{mV}$ (Fig. 3C, inset). Untreated astrocytes at all DOT displayed only outwardly rectifying, non-inactivating currents at membrane potentials more positive than $-40 \mathrm{mV}$ (Fig. 3B and C, left). Their voltage-dependent kinetics (Fig. 3E, F) and the partial inhibition by extracellular administration of $10 \mathrm{mM}$ TEA (data not shown) strongly suggest that they were mainly mediated by voltage-gated $\mathrm{K}^{+}$channels [21]. G5-treated astrocytes at 5-7 DOT exhibited an increase in membrane conductance (Fig. 3B, right). Both positive and negative fast activating, non-inactivating membrane currents were elicited in the entire range of voltage stimulation. The currents had a quasi-linear current-voltage (I-V) profile (Fig. 3D) and changed polarity $\left(V_{\text {rev }}\right)$ at $\sim-50 \mathrm{mV}$. At $12-16$ DOT, larger quasi-instantaneous, non-inactivating currents were evoked both at positive and negative membrane potentials (Fig. 3C, right). Currents had $V_{\text {rev }}$ at $\sim-70 \mathrm{mV}$ and exhibited an ohmic behavior (Fig. 3E). The increase in membrane conductance upon time of exposure to G5 was also evident when comparing the I-V relationships of G5-treated astrocytes at 5-7 and 12-16 DOT (Fig. S2). The analysis of the input resistance (Ri) from the linear portion of the I-V curves at negative voltages measuring the instantaneous current from -60 to $-80 \mathrm{mV}$ revealed that compared to untreated astrocytes, G5 exposure caused a significant decrement of $\mathrm{Ri}$ at all DOT windows (Fig. 3F). A decrease in Ri was also observed when comparing G5 astrocytes at 8-11 and 12-16 DOT.

Taken together, these results show that compared to control astrocytes, those morphologically differentiated upon long-term exposure to the chemical-defined medium containing G5 display an augment of the background membrane conductance mediated by the time-dependent increase in different membrane currents.

\section{Differential pharmacological modulation of membrane conductance expressed by G5-treated primary cortical astrocytes}

Since the experiments above suggested the differential expression of membrane currents that contribute to the background conductance depending on the period of astrocyte exposure to the G5-containing medium, we next addressed their pharmacological sensitivity. Previous work showed that cortical astrocytes morphologically differentiated upon long-term exposure to a membrane-permeable analog of cyclic-AMP (dibutyryl-cAMP, dBcAMP) expressed inward rectifier $\mathrm{K}^{+}$and $\mathrm{Cl}^{-}$currents that contribute to set the RMP and are specifically inhibited by extracellular exposure to micromolar concentrations of barium $\left(\mathrm{Ba}^{2+}\right)$ and cadmium $\left(\mathrm{Cd}^{2+}\right)$, respectively [21]. We hence addressed the ability of these ions to depress the currents expressed by G5-treated cultured astrocytes.

In Fig. 4 are shown the differential $\mathrm{Ba}^{2+}$ and $\mathrm{Cd}^{2+}$ sensitivities of the background conductance in astrocytes analyzed at the two most distant DOT windows. In 5-7 DOT G5 astrocytes, both positive and negative ramp currents elicited from -120 to $+60 \mathrm{mV}$ were not affected by extracellular $\mathrm{Ba}^{2+}(200 \mu \mathrm{M})$. By contrast negative currents were inhibited by subsequent co-administration of $\mathrm{Cd}^{2+}(200 \mu \mathrm{M})$ and $\mathrm{Ba}^{2+}$ (Fig. 4A, B). The voltage intercept of ramp currents before and after $\mathrm{Ba}^{2+}$ and $\mathrm{Cd}^{2+}$ co-administration was near the zero-current level. The same stimulation protocol revealed that in 12-16 DOT G5 astrocytes, $\mathrm{Ba}^{2+}$ caused a depression of the ramp current (Fig. 4C), which, however, was significant only at negative voltages and was not further attenuated by co-application of $\mathrm{Cd}^{2+}$ and $\mathrm{Ba}^{2+}$ (Fig. 4D). The voltage intercept of ramp currents before and after $\mathrm{Ba}^{2+}$ administration was at $\sim-85 \mathrm{mV}$ strongly suggesting that in these astrocytes, the resting conductance was mediated by a $\mathrm{Ba}^{2+}$-sensitive $\mathrm{K}^{+}$conductance. Overall, in this analysis, G5-treated astrocytes at 5-7 DOT with RMPs 
Fig. 3 Time-dependent effects of G5 challenge on whole-cell membrane conductance of primary cortical astrocytes. A Bar graph of mean membrane capacitance $(\mathrm{Cm})$ values in untreated (ctrl) and G5-treated astrocytes at the various DOT windows. $* p<0.05$ and $* * * p<0.001$ with one-way ANOVA followed by Bonferroni's multiple comparisons test. B Representative current families elicited with a family of 400-ms voltage steps from a holding potential $\left(V_{h}\right)$ of $-60 \mathrm{mV}$ and steps from -120 to $+60 \mathrm{mV}$ in $20-\mathrm{mV}$ increments (inset of C) in an 5-7 DOT untreated (ctrl) astrocyte (left) and in an astrocyte grown in G5 for the same DOT (right). C Representative current families at $12-16$ DOT untreated (left) and G5-treated astrocytes (right). Dashed lines in B and C are the zero-current levels. D Currentvoltage relationship (I-V) of mean steady-state current densities from 5 to 7 DOT untreated (ctrl, black, $n=13$ ) and 5-7 DOT G5 astrocytes (red, $n=12$ ). E I-V plot of 12-16 DOT untreated astrocytes (ctrl, black, $n=6$ ) and upon 12-16 DOT with G5 (red, $n=11) * p<0.05, * * p<0.01$, and $* * * p<0.001$ with unpaired Student's $t$-test. F Semi-logarithmic bar graph of the mean values of the input resistance in the two conditions at different DOT windows. $* p<0.05, * * p<0.01$, $* * * p<0.001$, and $\# p<0.05$ with one-way ANOVA followed by Bonferroni's multiple comparisons test
A

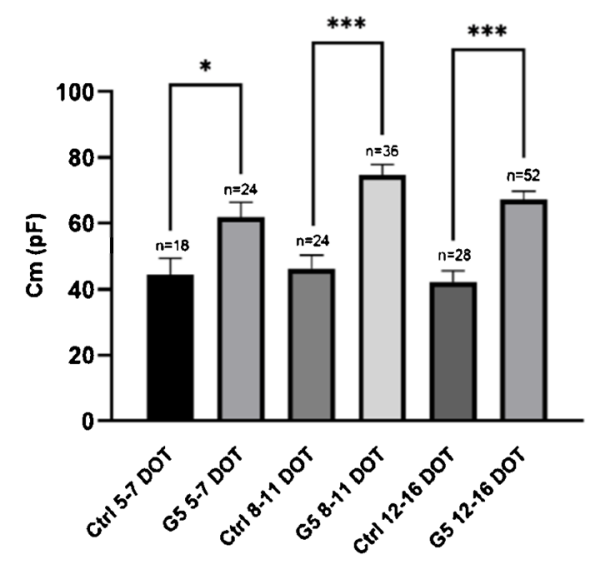

$\mathbf{F}$

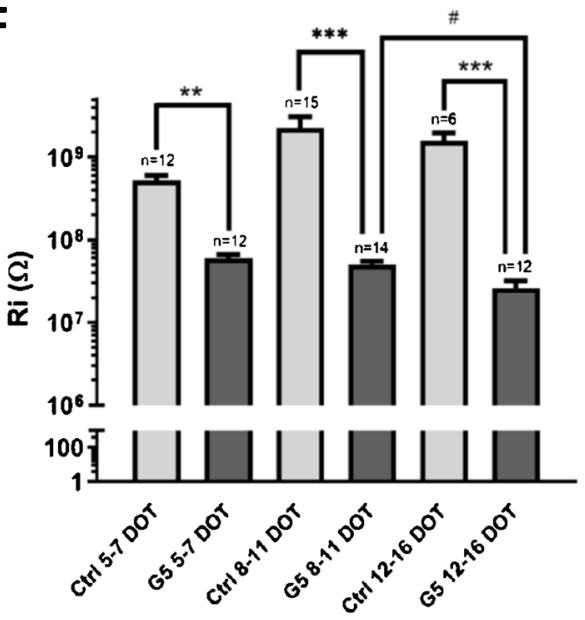

B

5-7 DOT

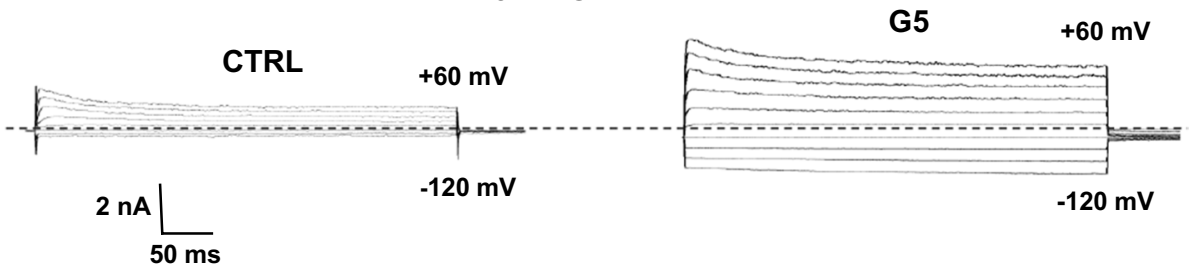

C

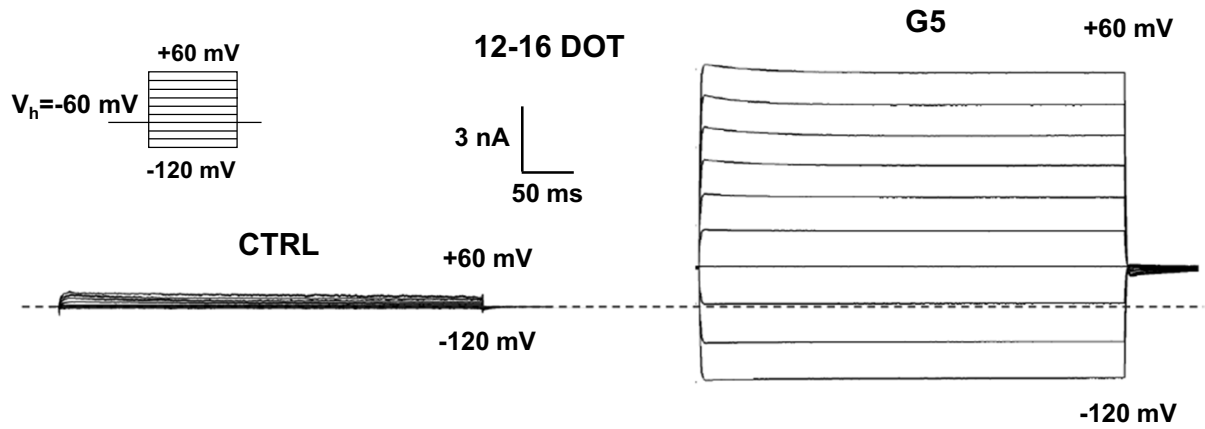

D

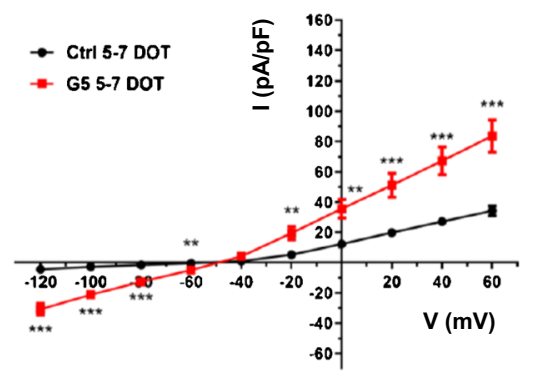

E

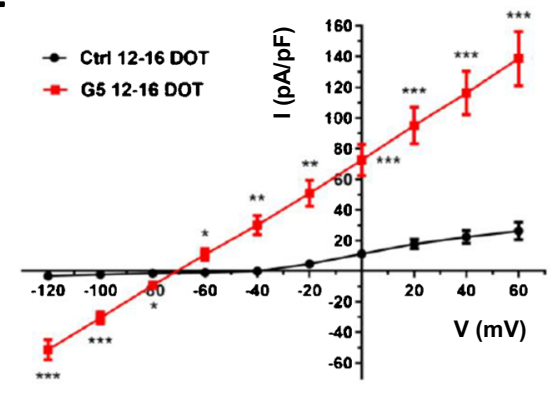

more positive than $-60 \mathrm{mV}$ ( 12 of 15 astrocytes) displayed the prevalence of $\mathrm{Cd}^{2+}$-sensitive current with a non-significant contribution of the $\mathrm{Ba}^{2+}$-sensitive $\mathrm{K}^{+}$ current. The opposite was observed in 12-16 DOT G5 astrocytes which had RMPs more negative than $-60 \mathrm{mV}$
(26 of 31 astrocytes). Altogether these findings confirm that astrocytes morphologically differentiated upon longterm exposure to G5 exhibit a time-dependent expression of background membrane currents with differential pharmacological sensitivity. 
A

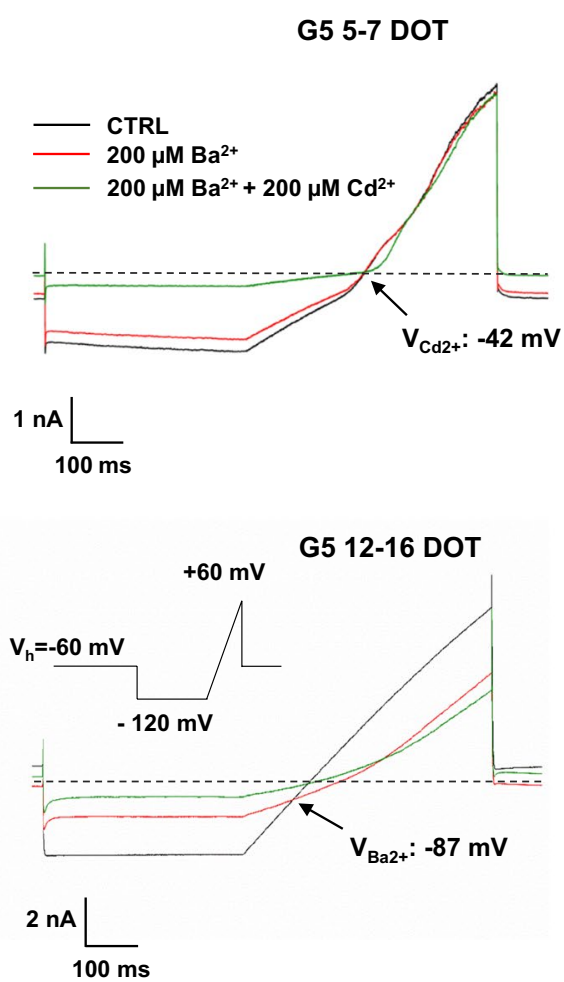

B

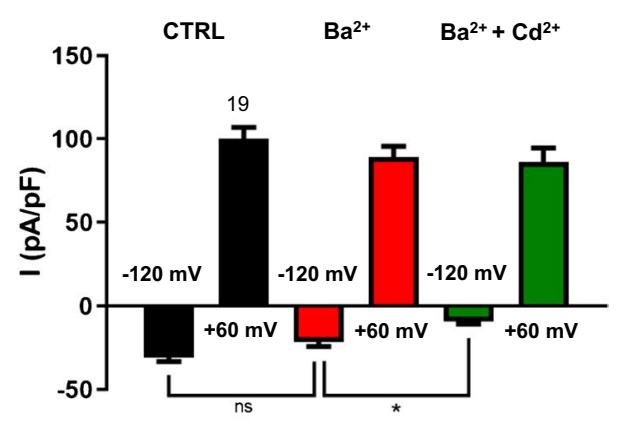

D

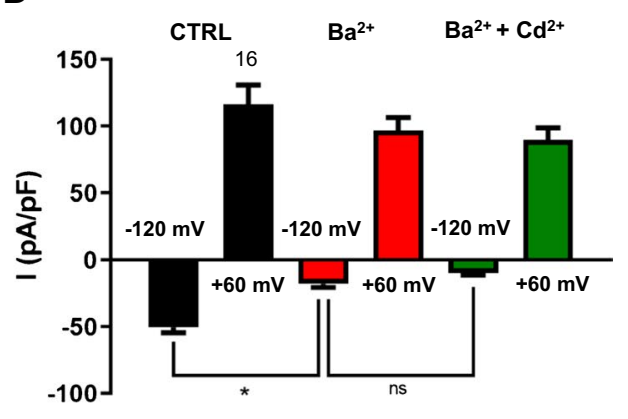

Fig. 4 Time-dependent differential pharmacological sensitivity of whole-cell membrane conductance in G5-treated primary cortical astrocytes. A Representative currents evoked in a 5-7 DOT G5 astrocyte with a ramp protocol (inset of C) stepping from a $V_{h}$ of -60 to $-120 \mathrm{mV}$ for $400 \mathrm{~ms}$ before applying a depolarizing ramp (180 $\mathrm{mV} / 500 \mathrm{~ms}$ ). Currents are those remaining stable for at least $2 \mathrm{~min}$ in control conditions (ctrl, black trace), following the maximal effect of barium administration $\left(\mathrm{Ba}^{2+}, 200 \mu \mathrm{M}\right.$, red trace) and upon the subsequent addition of cadmium $\left(\mathrm{Cd}^{2+}, 200 \mu \mathrm{M}\right)$ to the $\mathrm{Ba}^{2+}$-containing solution (green trace). The voltage intercept $\left(\mathrm{V}_{\mathrm{Cd} 2+}\right)$ of the currents before and after addition of $\mathrm{Cd}^{2+}$ was near the zero-current potential. Dashed line is the zero-current level. The RMP of this astrocyte was $-44 \mathrm{mV}$. B The bar graph of the quantitative analysis of the current densities at $+60 \mathrm{mV}$ and $-120 \mathrm{mV}$ in the absence and presence of $\mathrm{Ba}^{2+}$ and $\mathrm{Ba}^{2+}+\mathrm{Cd}^{2+}$ depicts a significant current diminution only at

\section{Identification of the ion currents expressed by G5-treated primary cortical astrocytes}

Since 12-16 DOT G5 astrocytes display an increase in $\mathrm{K}^{+}$ conductance, we next sought to identify the underlying channels. Cortical astrocytes in vitro express Kir-channelmediated currents under specific culture conditions $[2,21]$ and two-p-domain $\mathrm{K}^{+}(\mathrm{K} 2 \mathrm{P})$ channels that are activated by specific stimuli $[22,25,51]$. In vivo both channels contribute to the large background conductance [64, 94]. However, the high $\mathrm{Ba}^{2+}$ sensitivity of the $\mathrm{K}^{+}$conductance expressed by astrocytes upon G5 for 12-16 DOT diminished the probability of the contribution of K2P-mediated current because in cultured astrocytes, K2P channels are only slightly inhibited by higher $\mathrm{Ba}^{2+}$ concentration [22]. We hence hypothesized
$-120 \mathrm{mV}$ upon $\mathrm{Cd}^{2+}$ administration. ${ }^{*} p<0.05$ with one-way ANOVA followed by Bonferroni's multiple comparisons test. C Representative ramp current from a 12-16 DOT G5 astrocyte with a RMP of -76 $\mathrm{mV}$ and elicited with the same protocol as in A. The voltage intercept $\left(V_{\mathrm{Ba} 2+}\right)$ of the ramp currents before and after addition of $\mathrm{Ba}^{2+}$ is close to the Nernst equilibrium potential for potassium $\left(\mathrm{K}^{+}\right)$under the experimental conditions used. Dashed line is the zero-current level. D The bar graph of the quantitative analysis of the current densities at $+60 \mathrm{mV}$ and $-120 \mathrm{mV}$ in the absence and presence of $\mathrm{Ba}^{2+}$ and $\mathrm{Ba}^{2+}$ $+\mathrm{Cd}^{2+}$ shows that a significant current attenuation was observed only at $-120 \mathrm{mV}$. ${ }^{*} p<0.05$ with one-way ANOVA followed by Bonferroni's multiple comparisons test. Numbers above black bars in B and D depict the number of cells in each condition. Ns identifies non-significant differences

that the G5-induced $\mathrm{K}^{+}$conductance was mediated by Kir channels.

In cultured astrocytes, the kinetics of Kir current depend on extracellular $\mathrm{K}^{+}$concentration $\left(\left[\mathrm{K}^{+}\right]_{\mathrm{o}}\right)$ that yields an increase in chord conductance at potentials more negative than $V_{\text {rev }}[2,71]$. We analyzed the ramp current kinetics in physiological $\left[\mathrm{K}^{+}\right]_{\mathrm{o}}$ and upon a tenfold increase. The results show that at 5-7 DOT upon G5, the change in $\left[\mathrm{K}^{+}\right]_{\mathrm{o}}$ from 4 to $40 \mathrm{mM}$ caused a positive shift of $\sim 20 \mathrm{mV}$ in $V_{\text {rev }}$ but did not affect the chord conductance (Fig. 5A, B). By contrast, at 12-16 DOT with G5, the same experimental protocol induced a $\sim 50 \mathrm{mV}$ positive shift of $V_{\text {rev }}$ and an increase in $\mathrm{Ba}^{2+}$-sensitive chord conductance at potentials negative to $V_{\text {rev }}$ (Fig. 5C, D). These results support the view that cultured astrocytes grown for long-term in G5-containing 
A

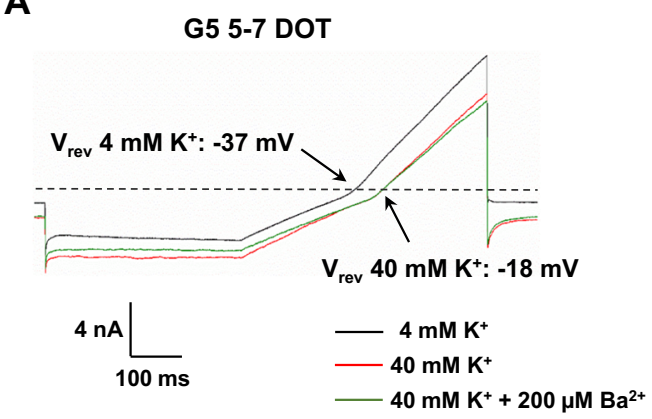

C

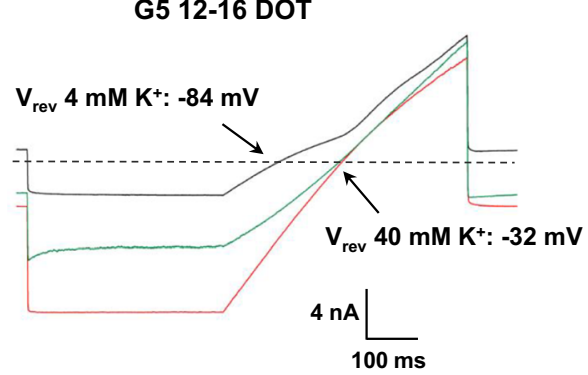

B

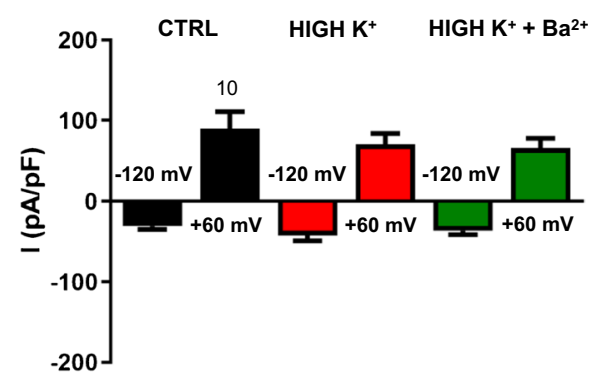

D

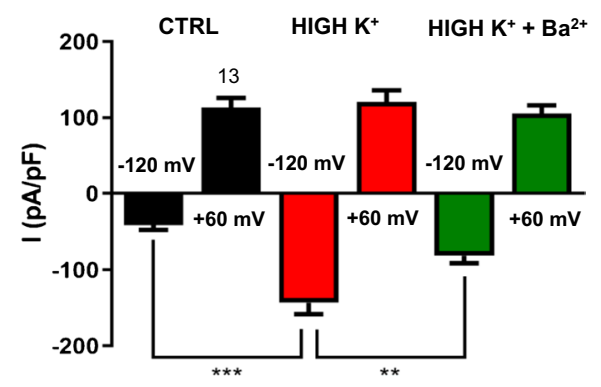

$+\mathrm{Ba}^{2+}$ ) in 5-7 G5 astrocytes. C Representative currents evoked in a 12-16 DOT G5 astrocyte with the same protocol as in A. The tenfold increase in extracellular $\mathrm{K}^{+}$induced a $\sim 50 \mathrm{mV}$ positive shift in current reversal potential, an increase in membrane conductance at ramp potentials more negative than the reversal potential, and the partial depression by $\mathrm{Ba}^{2+}$ administration. Dashed line is the zero-current level. D The quantitative analysis shows that the ten-fold increase in extracellular $\mathrm{K}^{+}$enhanced the current magnitude only at $-120 \mathrm{mV}$, which was partially attenuated by $\mathrm{Ba}^{2+}$ administration. ${ }^{*} p<0.01$ and $* * * p<0.001$ with one-way ANOVA followed by Bonferroni's multiple comparisons test. Numbers above black bars in B and D depict the number of cells in each condition

these ionic conditions, 5-7 DOT G5 astrocytes exhibited large strongly inward-rectifying currents which activated slowly at step potentials more negative than $-20 \mathrm{mV}$ and did not show voltage dependent inactivation (Fig. 6A). The hyperpolarization-activated conductance was significantly attenuated by submillimolar $\mathrm{Cd}^{2+}$ (Fig. 6B, E) corroborating the tenet that it was mediated by the inwardly rectifying $\mathrm{Cl}^{-}$current previously identified in cultured astrocytes [20, 21, 45]. In 12-16 DOT G5 astrocytes, the amplitude of the $\mathrm{Cl}^{-}$conductance was larger (Fig. 6C, D) owing to an increase in current density (Fig. 6F).

Altogether, these results indicate that the morphological differentiation of long-term G5-treated astrocytes is accompanied by the early expression of an inward rectifier $\mathrm{Cl}^{-}$current, which precedes the functional appearance of the inward rectifier $\mathrm{K}^{+}$current. 


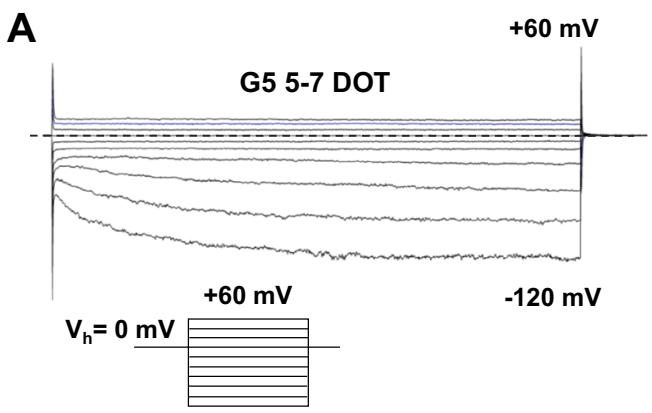

$-120 \mathrm{mV}$
B

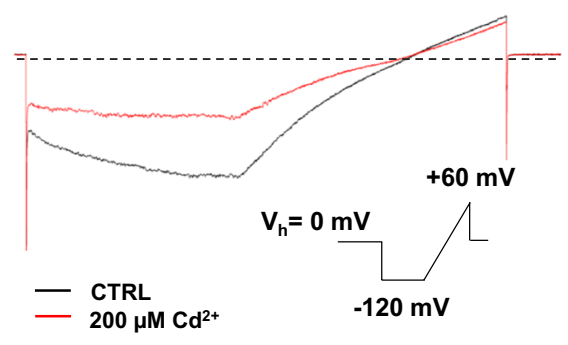

D

C

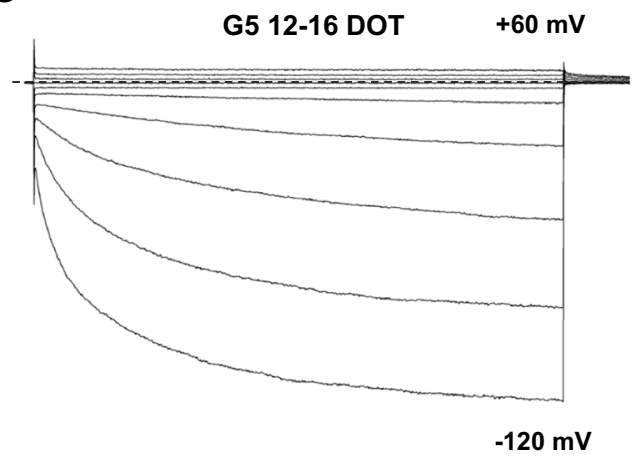

E

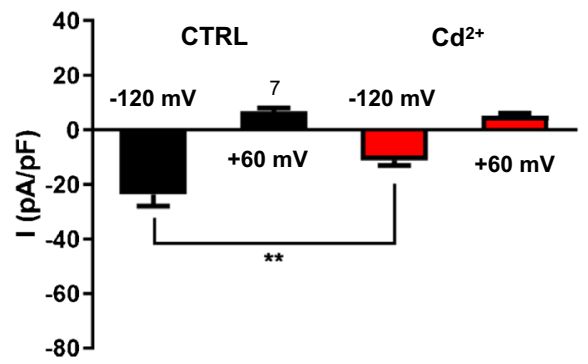

Fig. 6 An inward rectifier $\mathrm{Cl}^{-}$current underlies the cadmiumsensitive conductance expressed by G5-treated primary cortical astrocytes.A Representative familyof currents elicited in a 5-7 DOT G5 astrocyte with a voltage steps protocol (inset) consisting of 2-s long voltage steps from a $V_{h}$ of $0 \mathrm{mV}$ applying step potentials from -120 to $+60 \mathrm{mV}$ in $20 \mathrm{mV}$ increments and measured using intra- and extra-cellular solutions with symmetrical high chloride $\left(\mathrm{Cl}^{-}\right)$and the monovalent cations replaced with cesium. Time-dependent currents activated slowly and were strongly inwardly rectifying. B The slow ramp current $(180 \mathrm{mV} / 1 \mathrm{~s})$ activated following a voltage step of 800 $\mathrm{ms}$ to $-120 \mathrm{mV}$ from a $V_{h}$ of $0 \mathrm{mV}$ (inset) in the same astrocyte of A was markedly depressed by extracellular $\mathrm{Cd}^{2+}(200 \mu \mathrm{M}$, red trace),

\section{Molecular identification of the channel proteins mediating the increased membrane conductance in G5-treated primary cortical astrocytes}

The functional analyses supported the view that astrocytes morphologically differentiated upon long-term G5
$\mathbf{F}$

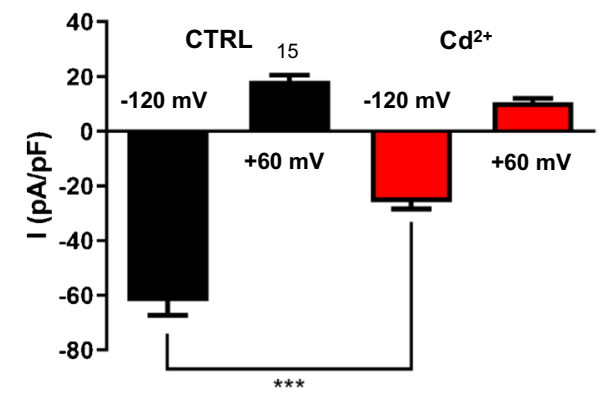

which inhibits inward rectifier $\mathrm{Cl}^{-}$channels. C Representative voltage-step currents in a 12-16 DOT G5 astrocyte recorded in the same conditions as in A are grater. D The ramp current in the same astrocyte retained the partial inhibition by $\mathrm{Cd}^{2+}$. Scale bars are the same for all current traces. Dashed lines represent the zero-current level. E-F Bar graph of ramp current densities of the inward rectifier $\mathrm{Cl}^{-}$ current at +60 and $-120 \mathrm{mV}$ measured in astrocytes exposed to $\mathrm{G} 5$ for 5-7 DOT (E) and 12-16 DOT (F) in the absence (ctrl) and following the maximal inhibition upon extracellular administration of $\mathrm{Cd}^{2+}$ $(200 \mu \mathrm{M}) .{ }^{* *} p<0.01$ and $* * * p<0.001$ with paired Student's $t$-test. Numbers above bars depict the number of cells in each condition

exposure express inward rectifier $\mathrm{Cl}^{-}$and $\mathrm{K}^{+}$currents. To determine whether these results were associated with an increment in ion-channel protein synthesis, we performed immunocytochemical and immunoblotting analyses in 12-16 DOT astrocytes in the absence and presence of G5. The functional analysis suggested that the increase 
Fig. 7 Variations in molecular expression of chloride and potassium channels in G5-treated primary cortical astrocytes.A-B Confocal analyses on sub-confluent astrocytes co-immunostained for glial fibrillary acidic protein (GFAP, red) and $\mathrm{ClC}-2$ protein (green). ClC-2 is undetectable in 14 DOT untreated GFAP-positive astrocytes (A) but is present in 14 DOT G5 astrocytes (B). Merge image depicts partial signal overlay. C-D Representative western blot (C) of ClC-2 monomer from total lysates of untreated (ctrl) and 12-16 DOT G5 astrocytes with relative densitometric analysis $(\mathbf{D}, n=3)$.EF Confocal analyses performed on sub-confluent astrocytes coimmunostained for GFAP (red) and Kir4.1 (green). Kir4.1 staining is negligible in untreated GFAP-positive astrocytes (ctrl, E) and strongly upregulated in 14 DOT G5 astrocytes $(\mathbf{F})$. Merge image depicts partial signal overlay. Scale bars in A and E: $20 \mu \mathrm{m}$. G-H Representative western blot $(\mathbf{G})$ of $\mathrm{Kir} 4.1$ monomer from total lysates of untreated (ctrl) and 12-16 DOT G5-treated astrocytes with relative densitometric analysis $(\mathbf{H}$, $n=3)$. $* * p<0.01$ with unpaired Student's $t$-test
A

CTRL (14 DOT)

B G5 (14 DOT)

C

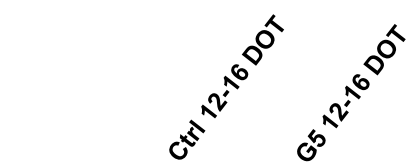

$100 \mathrm{kDa}$

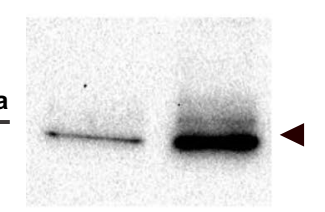

CIC-2 IB

$37 \mathrm{kDa}$

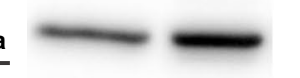

$\beta$-actin IB

E

CTRL (14 DOT)

$\mathbf{F}$

G5 (14 DOT)
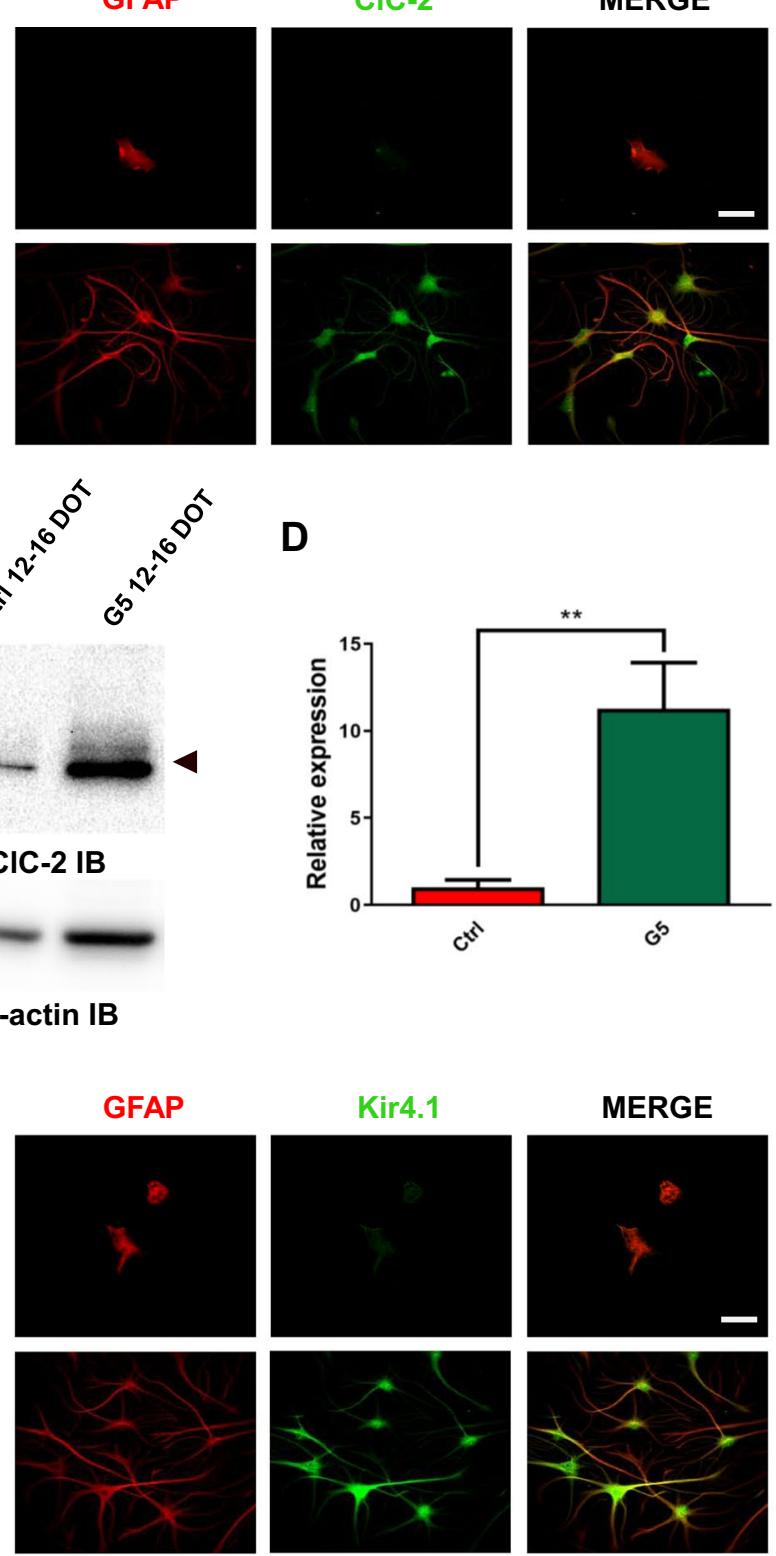

D
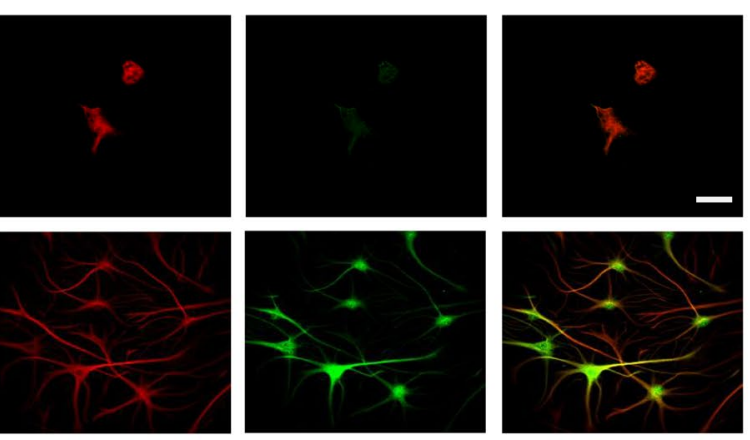

G

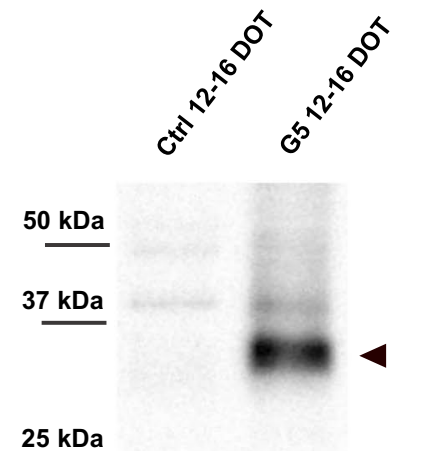

Kir4.1 IB

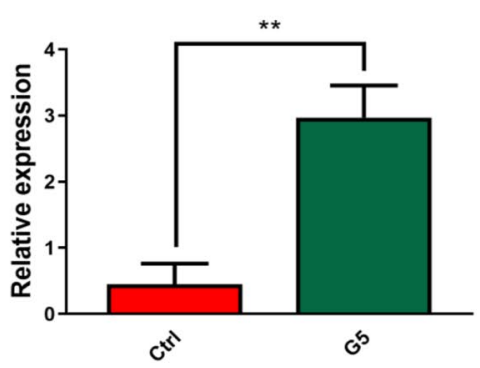

H<smiles></smiles><smiles>C1CCC1</smiles> 
Fig. 8 Changes in expression of intermediate filament proteins in G5-treated primary cortical astrocytes.A)Representative western blot of GFAP and vimentin from total lysates of untreated (ctrl) and 12-16 DOT G5 astrocytes. B-C) The densitometric analyses $(n=3$ for each condition) show the marked diminution in GFAP and vimentin levels upon prolonged G5 treatment. ** $p<0.01$ with unpaired Student's $t$-test

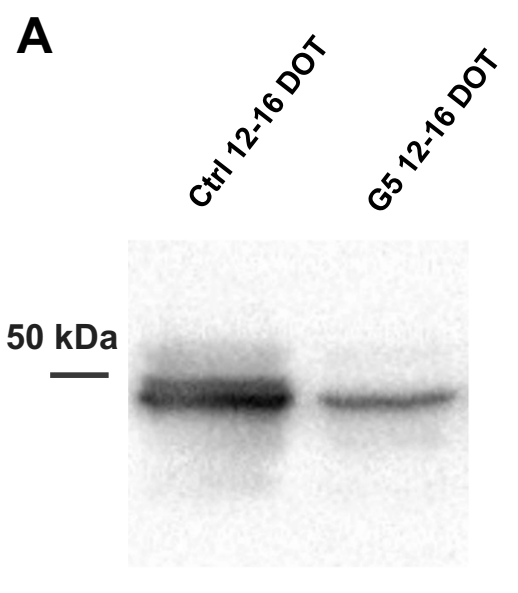

GFAP IB

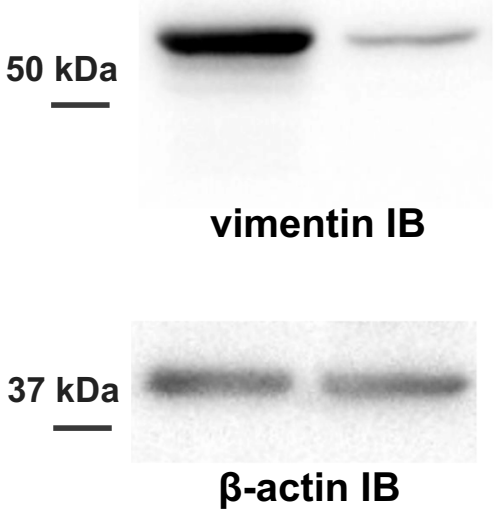

B

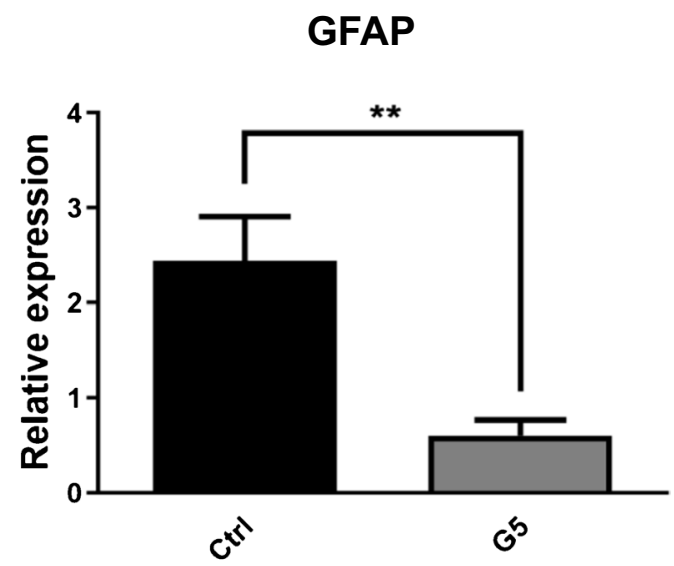

C

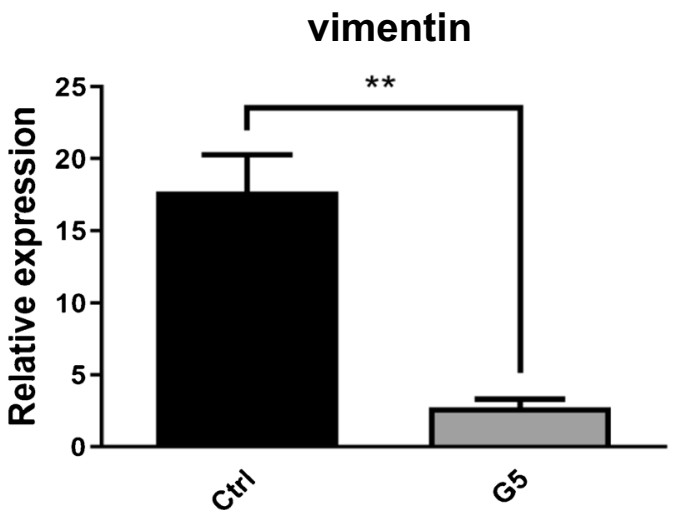

in $\mathrm{Cl}^{-}$conductance was mediated by the upregulation of ClC-2, which was shown to be functionally expressed in astroglial cells both in vitro and in vivo[36, 46]. The results obtained by confocal analysis in untreated (ctrl) and G5-treated sub-confluent astrocytes at 14 DOT show that $\mathrm{ClC}-2$ (green) was highly expressed both in cell body and in multi-branched processes of GFAP-positive cells (red) but was virtually undetectable in untreated astrocytes (Fig. 7A, B). Immunoblotting analysis revealed that in lysates of long-term G5-treated astrocytes, the expression level of ClC-2 monomer was tenfold higher compared to that of untreated cells (Fig. 7C, D). Since the ClC-2-mediated currents developed early upon G5 treatment, we also assessed the protein expression in whole-cell lysates of astrocytes at 5-7 DOT. Surprisingly, we did not observe any difference in total protein expression between untreated astrocytes and those G5-treated (Fig. S3).

We next characterized the expression pattern of the Kir4.1 channel, which previous studies identified to be the predominant Kir channel protein expressed in astrocytes from different regions in vivo [29, 31, 42, 60, 75] but also under some culture conditions in vitro [2]. The immunofluorescence analysis of untreated (ctrl) and 14 DOT G5-treated astrocytes revealed that in GFAP positive cells (red), $\mathrm{K}_{\mathrm{ir}} 4.1$ expression (green) was not detectable in control astrocytes but was diffusely present in G5-treated astrocytes (Fig. 7E, F). Qualitatively similar results were obtained by comparing the immunoblot determinations in the two conditions which show that in whole-cell protein extracts, the monomeric form of Kir4.1 was increased by $\sim$ sevenfold (Fig. 7G, H).

\section{G5-treated primary cortical astrocytes display a non-reactive biochemical phenotype}

Since changes in morphology denoted by the appearance of process elongation is also observed in reactive astrocytes [18], we evaluated whether the morphological differentiation induced by $\mathrm{G} 5$ challenge was associated to an increase in GFAP expression, which is a typical hallmark of reactive astrocytes [17]. The immunoblot analysis shows that compared to untreated astrocytes, those exposed to G5 for 12-16 DOT exhibited a robust diminution of GFAP expression (Fig. 8A, $\mathrm{B})$, and the same result was observed for the other intermediate 
filament vimentin (Fig. 8A, C), which is also upregulated in reactive astrocytes [66]. Altogether, this result suggests that the long-term culturing of primary rat cortical astrocytes in G5-containing chemically defined medium promotes the growth of non-reactive, quiescent astrocytes that display functional features observed in astrocytes in vivo.

\section{Discussion}

The main objective of this study was to determine the long-term functional consequences of the grown of primary cortical astrocytes in a chemical-defined medium supplemented with a cocktail (G5) of growth factors and hormones that previous studies reported to cause their in vitro activation and morphological differentiation $[8$, $27,86,87]$. We show that the long-term G5 challenge caused the gradual, time-dependent morphological differentiation of the astrocytes that acquire a multi-branched, process-bearing phenotype accompanied by variations of passive membrane properties and by time-dependent differential expression of $\mathrm{K}^{+}$and $\mathrm{Cl}^{-}$membrane channels involved in the homeostatic functions of astrocytes.

Usually, cultured astrocytes are grown in a medium containing 10\% FBS which allows rapid proliferation [47]. At confluence FBS-grown cortical astrocyte monolayer has a cobblestone phenotype, and re-plated isolated astrocytes display a fibroblast-like, epithelioid morphological phenotype [54]. In our experimental model, FBS was gradually omitted when astrocytes reached confluence ( 10 days), and re-plated astrocytes in the absence of FBS were supplemented with G5 for up to 2 weeks thereafter. During this period, astrocytes gradually changed their shape displaying polarized processes departing from the cell body after 5-7 DOT and acquiring a multipolar, fine-branched phenotype with a retracted cell body at $12-16$ DOT. These morphological alterations resemble those observed in long-term cultured astrocytes grown in various chemically defined medium [54]. Similar results have also been obtained with a method in which immunopanning of microglia, macroglia, and neurons was used to culture astrocytes in FBS-free, heparin-binding epidermal growth factor (EGF)-containing medium [23, 92]. However, it is worth noting that also in those approaches, FBS was used in some purification steps of the astrocyte cultures. Recently, a method using a FBSfree, chemically defined medium containing fibroblast growth factor-2 (FGF2) and epidermal growth factor (EGF) was shown to promote the growth of mice-cultured astrocytes with morphological and biosynthetic features similar to those of in vivo astrocytes and characterized, compared to untreated cells, by a process-bearing morphology and an increase of glutamate uptake [70]. Of note, even though FGF and EGF are present in G5 cocktail, compared to untreated astrocytes, 12-16 DOT G5 astrocytes displayed a slight decrease in expression of the principal glutamate transporter GLT-1 (Fig. S4). Whether this negative modulation is due to the cellular morphological rearrangement or an antagonistic effect of other components of G5 administered in the absence of FBS remains to be established.

Our study clearly shows that the morphological differentiation of G5-treated cortical astrocytes was paralleled by variations in membrane conductance. The results indicate that G5 challenge for 5-7 DOT caused a marked decrease in membrane resistance, which, however, compared to untreated astrocytes, was not accompanied by a significant change in RMP. The electrophysiological and pharmacological analyses revealed that the increase in membrane conductance was due, at least in part, to an increase in functional expression of the $\mathrm{Cl}^{-}$channel $\mathrm{ClC}-2$, which started early during G5 treatment and increased throughout the whole period of exposure. Notably, the upregulation of ClC-2-mediated $\mathrm{Cl}^{-}$inward rectifier at 5-7 DOT was not paralleled by an increment in total protein level. This discrepancy could be due to a G5-mediated augment in trafficking of ClC-2 from a cytosolic pool to the plasma membrane. In this context, previous work in cultured astrocytes identified significant amount of ClC-2 in the Golgi complex [78]. Moreover, cell surface expression and function of $\mathrm{ClC}-2$ was shown to be upregulated by microtubule perturbation [14]. Whether G5 treatment induces the early effect on ClC-2-mediated conductance through changes in microtubule-associated proteins that regulate astrocyte differentiation [13] remains to be established. At variance, G5 challenge for 12-16 DOT also upregulated newly synthesized $\mathrm{ClC}-2$ proteins, a result that was mirrored by a further increase in $\mathrm{Cl}^{-}$inward rectifier.

$\mathrm{ClC}-2$ is a member of the large family of $\mathrm{Cl}^{-}$channels that in mammals consist of 9 subtypes [35]. ClC-2 is expressed in various cell preparations and is highly enriched in the brain $[10,81]$. It is inhibited by submillimolar concentrations of $\mathrm{Cd}^{2+}$ and $\mathrm{Zn}^{2+}$ and allows $\mathrm{Cl}^{-}$outflow at membrane potentials below the equilibrium potential for $\mathrm{Cl}^{-}$[37]. Astrocytes express a variety of $\mathrm{Cl}^{-}$channels $[16,87]$. ClC-2 was shown to be expressed in astrocytes both in vitro and in situ [36, $46,77]$. In brain slices, ClC- 2 is localized at terminals of astrocyte processes abutting GABAergic synapses in the hippocampus [77]. An inward rectifier $\mathrm{Cl}^{-}$current with pharmacological and biophysical properties identical to $\mathrm{ClC}-2$ was reported to be expressed in cultured rat cortical astrocytes long-term treated with $\mathrm{dBcAMP}$ to induce their morphological differentiation $[20,21]$ and in astrocytes co-cultured with neurons [45]. The result that the functional expression of the inward rectifier $\mathrm{Cl}^{-}$conductance mediated by $\mathrm{ClC}-2$ is an early event associated to the morphological differentiation of the primary astrocytes induced by G5 exposure raises the question of whether this channel could play a role in the regulation of the differentiation process. Interestingly, in situ 
$\mathrm{ClC}-2$ is absent in morphologically immature astrocytes and in astrocytes that display morphological alterations within a brain lesion [46]. However, since reverse causality cannot be ruled out, further studies are warranted to address this issue.

The role of ClC-2 in astrocytes as well as in other cell types is still largely elusive [34]. Genetic ablation of ClC-2 did not cause overt defects in brain structural organization except widespread vacuolization of the white matter and spinal cord typical of leukoencephalopathy [4]. The absence of $\mathrm{ClC}-2$ has been associated with progressive neurodegeneration in old mice a result that was interpreted as due to astrocyte activation leading to defect in neurotransmission [12]. Recent works in cultured astrocytes indicate that $\mathrm{ClC}-2$ assembles with auxiliary subunits that change its biophysical properties [36, 78]. Those findings suggested that $\mathrm{ClC}-2$ also might mediate $\mathrm{Cl}^{-}$influx necessary to compensate the $\mathrm{K}^{+}$dynamics. Overall, the results of this study indicate that G5-treated cultured astrocytes could be a suitable experimental model for getting more insights on the functional impact of ClC-2 in astrocyte biology.

Our data also show that the ohmic profile of the large membrane conductance of cortical astrocytes exposed to G5 for 12-16 DOT is partially mediated by the upregulation of the inward rectifier $\mathrm{K}^{+}$channel Kir4.1. Kir4.1 is a member of the large family of Kir channels composed of 15 subtypes [30]. It is also the major component of the large astrocytic $\mathrm{K}^{+}$conductance and promotes the very negative RMP of astrocytes in vivo [15, 38, 60, 71, 75]. Kir4.1 is crucially involved in the regulation of $\left[\mathrm{K}^{+}\right]_{\mathrm{o}}[9,15,39]$ and to augmenting the efficacy of glutamate uptake $[15,33]$. In cultured astroglia, the evidence are more contradictory with reports indicating the presence of Kir4.1 [39, 60] and others failing to detect its functional expression [3, 21, 79]. These discrepancies could be related to the culture conditions and/ or the region of the CNS from which the tissues to prepare the culture were obtained. In this respect, it is well known that in situ Kir4.1 expression is heterogeneous throughout brain regions and in the spinal cord $[53,57,59,69,80]$. The current study confirms that under our experimental conditions and in the absence of G5, cultured cortical astrocytes do not display significant Kir4.1-mediated current. Even at $16 \mathrm{DOT}$, only outward rectifier $\mathrm{K}^{+}$current insensible to bath application of submillimolar $\mathrm{Ba}^{2+}$ could be detected in untreated astrocytes. By contrast in astrocytes at 8-11 DOT with G5, there was an increase in the proportion of astrocytes with a very negative RMP and an overall decrease in input resistance with a further decrement at 12-16 DOT. These results, together with the marked depression of the negative currents by $\mathrm{Ba}^{2+}$ at a concentration that selectively inhibits Kir4.1, suggest that G5 exposure promotes the gradual functional appearance of Kir4.1. This finding was corroborated by the observation that G5 challenge for 12-16 DOT induced a strong increase in Kir4.1 protein expression when compared to untreated cultures. Of note, compared to untreated astrocytes, the challenge with G5 at 5-7 and 12-16 DOT also caused an increment of positive current activated at depolarized membrane potentials. The increase in $\mathrm{K}^{+}$outflow through Kir4.1 could only partially explain the increase in positive current at 12-16 DOT because the upregulation was already observed in astrocytes at 5-7 DOT when Kir4.1 was not functionally expressed and $\mathrm{Ba}^{2+}$ application had minimal effect on outward current. However, the observation that this positive conductance was totally abolished when intracellular $\mathrm{K}^{+}$was replaced with the $\mathrm{K}^{+}$-channel impermeant cation $\mathrm{Cs}^{+}$suggests that it was mediated by an increase in $\mathrm{K}^{+}$current. Noteworthy, the upregulation of outward $\mathrm{K}^{+}$conductance could contribute to the consistent negative RMP observed in 5-7 G5 astrocytes in the presence of the depolarizing effect of the $\mathrm{Cl}^{-}$inward rectifier under our experimental conditions.

It has been reported that G5 challenge also promotes an increase saxitoxin-sensitive voltage-gated $\mathrm{Na}^{+}$channels [90]. The results of previous research [21] and of the current study do not support the presence of voltage-dependent $\mathrm{Na}^{+}$currents in untreated astrocytes. However, whether G5 affects the transcripts and/or whole-cell protein levels of the various $\mathrm{Na}^{+}$channels isoforms identified in different astrocyte preparations remains to be established [63].

Previous works showed that cultured astrocytes challenged with G5 displayed an increment of glutamate uptake capability linked to the increased expression of glutamate transporters [84-86]. However, whether that effect is influenced by additional mechanisms is unknown. The functional upregulation of Kir4.1 could partially explain the rise in glutamate uptake observed in G5-treated cultured astrocytes. A direct correlation between Kir4.1 expression and glutamate uptake has been demonstrated as in vivo the genetic ablation of Kir4.1 is associated to a decrement in glutamate uptake mediated by the $\mathrm{Na}^{+}$-dependent cotransporter GLT-1 [15]. Similar results have been described in cultured astrocytes in which Kir4.1 expression was downregulated by RNA interference [39]. It remains to be ascertained the effect on glutamate uptake of the large increase in Kir4.1 current in long-term G5-treated cortical astrocytes particularly in the context of the concomitant small diminution in total expression of GLT-1 transporter observed under these conditions. Whether the increase in expression of the $\mathrm{Cl}^{-}$channel ClC-2 plays a role in the regulation of glutamate uptake also warrants further investigations.

Primary cultured astrocytes have been often used for studying the astrocyte properties in physiological as well as pathological contexts [40, 49]. We and others have previously shown that incubation of cultured cortical astrocytes with the cell permeable cAMP analog dBcAMP caused morphological and biochemical changes $[21,43,76,89]$. The adherence of that culture model to 
physiological or pathological conditions in vivo is still uncertain $[19,62,88]$, and therefore, better in vitro models are required. The results of this study support the view that the long-term astrocyte culturing in a chemically defined medium containing G5 in the absence of FBS produces changes in astrocyte morphology and the acquisition of a quiescent phenotype since 12-16 DOT with G5 caused a strong downregulation in the expression of the intermediate filaments GFAP and vimentin, which are upregulated in reactive astrocytosis in vivo $[41,65,73]$. This observation contrasts with previous work in which few days of G5 challenge caused the upregulation of GFAP and vimentin [27]. The reason for this discrepancy is unclear but the fact that in our study G5 challenge was performed in the absence of FBS could play a role. To corroborate this hypothesis, it was previously reported that FBS affects several properties of cultured astrocytes [11]. Moreover, the fact that the G5 challenge was prolonged up to 2 weeks could also be a plausible explanation. Altogether, these results indicate that a supplement containing growth factors and hormones such as those present in G5 cocktail supports the long-term growth of primary cultured astrocytes in the absence of FBS and induce their morphological and functional differentiation by upregulating relevant homeostatic channel proteins.

\section{Conclusions}

Even though major advances in the understanding about the role of astrocytes in the physiology and pathophysiology of the CNS has been obtained from studies performed in primary cultured astrocytes, in the last decades, it has become clear that in vitro models not always mimic perfectly the complex situation occurring in vivo [40]. Despite this evidence, the use of a reductionist model such as primary cultures is still essential to address the contribution of specific stimuli in determining the dynamics of astrocyte plasticity under controlled conditions. In this context in the last years, great effort has been made to define conditions that more closely resembles those in vivo $[23,74]$. The results of this study add another piece of evidence to the view that the manipulation of culture conditions through the addition of specific growth factors and hormones could be used to create a valid in vitro platform to investigate the modulatory processes of homeostatic functions dependent on the expression of plasma membrane ion channels.

Supplementary Information The online version contains supplementary material available at https://doi.org/10.1007/s00424-021-02627-x.
Acknowledgements The authors thank Alessia Minardi for astrocyte culture preparation and Cecilia Franciosi for contributing to biochemical experiments.

Author contribution Francesco Formaggio and Martina Fazzina conducted experiments and performed data analysis. Raúl Estévez and Marco Caprini provided resources and helped with analysis. Francesco Formaggio and Stefano Ferroni designed the study. Francesco Formaggio and Stefano Ferroni wrote the paper.

Funding Open access funding provided by Alma Mater Studiorum Università di Bologna within the CRUI-CARE Agreement. This work was supported by RFO2018 to SF and RFO2017 to MC and by the Spanish Ministerio de Ciencia e Innovación (MICINN) (RTI2018093493-B-I00) to RE. FF was supported by University of Bologna (Assegno di Ricerca 537/2019). Part the experiments were used to partially fulfill the master thesis of MF.

\section{Declarations}

Conflict of interest The authors declare no competing interests.

Open Access This article is licensed under a Creative Commons Attribution 4.0 International License, which permits use, sharing, adaptation, distribution and reproduction in any medium or format, as long as you give appropriate credit to the original author(s) and the source, provide a link to the Creative Commons licence, and indicate if changes were made. The images or other third party material in this article are included in the article's Creative Commons licence, unless indicated otherwise in a credit line to the material. If material is not included in the article's Creative Commons licence and your intended use is not permitted by statutory regulation or exceeds the permitted use, you will need to obtain permission directly from the copyright holder. To view a copy of this licence, visit http://creativecommons.org/licenses/by/4.0/.

\section{References}

1. Barres BA, Koroshetz WJ, Chun LLY, Corey DR (1990) Ion channel expression by white matter glia: the type-1 astrocyte. Neuron 5:527544. https://doi.org/10.1016/0896-6273(90)90091-S

2. Benfenati V, Caprini M, Nobile M, Rapisarda C, Ferroni S (2006) Guanosine promotes the up-regulation of inward rectifier potassium current mediated by Kir4.1 in cultured rat cortical astrocytes. J Neurochem 98:430-445. https://doi.org/10.1111/j. 1471-4159.2006.03877.x

3. Bevan S, Raff M (1985) Voltage-dependent potassium currents in cultured astrocytes. Nature 315:229-232. https://doi.org/10. $1038 / 315229 \mathrm{a} 0$

4. Blanz J, Schweizer M, Auberson M, Maier H, Muenscher A, Hübner CA, Jentsch TJ (2007) Leukoencephalopathy upon disruption of the chloride channel ClC-2. J Neurosci 27:65816589. https://doi.org/10.1523/JNEUROSCI.0338-07.2007

5. Bordey A, Lyons SA, Hablitz JJ, Sontheimer H (2001) Electrophysiological characteristics of reactive astrocytes in experimental cortical dysplasia. J Neurophysiol 85:1719-1731. https://doi.org/10.1152/jn.2001.85.4.1719

6. Butt AM, Kalsi A (2006) Inwardly rectifying potassium channels (Kir) in central nervous system glia: a special role for Kir4.1 in glial functions. J Cell Mol Med 10:33-44. https://doi. org/10.1111/j.1582-4934.2006.tb00289.x 
7. Carbone M, Duty S, Rattray M (2012) Riluzole elevates GLT-1 activity and levels in striatal astrocytes. Neurochem Int. https:// doi.org/10.1016/j.neuint.2011.10.017

8. Carbone M, Duty S, Rattray M (2012) Riluzole neuroprotection in a Parkinson's disease model involves suppression of reactive astrocytosis but not GLT-1 regulation

9. Chever O, Djukic B, McCarthy KD, Amzica F (2010) Implication of Kir4.1 channel in excess potassium clearance: an in vivo study on anesthetized glial-conditional Kir4.1 knock-out mice. J Neurosci 30:15769-15777. https://doi.org/10.1523/JNEUR OSCI.2078-10.2010

10. Cid LP, Montrose-rafizadeh C, Smith DI, Guggino WB, Cutting GR (1995) Cloning of a putative human voltage-gated chloride channel (CIC-2) cDNA widely expressed in human tissues. Hum Mol Genet 4:407-413. https://doi.org/10.1093/hmg/4.3. 407

11. Codeluppi S, Gregory EN, Kjell J, Wigerblad G, Olson L, Svensson CI (2011) Influence of rat substrain and growth conditions on the characteristics of primary cultures of adult rat spinal cord astrocytes. J Neurosci Methods 197:118-127. https://doi.org/10. 1016/j.jneumeth.2011.02.011

12. Cortez MA, Li C, Whitehead SN, Dhani SU, D'Antonio C, Huan LJ, Bennett SAL, Snead OC, Bear CE (2010) Disruption of $\mathrm{ClC}-2$ expression is associated with progressive neurodegeneration in aging mice. Neuroscience 167:154-162. https://doi.org/ 10.1016/j.neuroscience.2010.01.042

13. Couchie D, Fages C, Bridoux AM, Rolland B, Tardy M, Nunez $\mathrm{J}$ (1985) Microtubule-associated proteins and in vitro astrocyte differentiation. J Cell Biol 101:2095-2103. https://doi.org/10. 1083/jcb.101.6.2095

14. Dhani SU, Mohammad-Panah R, Ahmed N, Ackerley C, Ramjeesingh M, Bear CE (2003) Evidence for a functional interaction between the $\mathrm{ClC}-2$ chloride channel and the retrograde motor dynein complex. J Biol Chem. https://doi.org/10.1074/ jbc.M209828200

15. Djukic B, Casper KB, Philpot BD, Chin LS, McCarthy KD (2007) Conditional knock-out of Kir4.1 leads to glial membrane depolarization, inhibition of potassium and glutamate uptake, and enhanced short-term synaptic potentiation. J Neurosci 27:1135411365. https://doi.org/10.1523/JNEUROSCI.0723-07.2007

16. Elorza-Vidal X, Gaitán-Peñas H, Estévez R (2019) Chloride channels in astrocytes: structure, roles in brain homeostasis and implications in disease. Int J Mol Sci 20:1034-1057. https://doi.org/10. 3390/ijms20051034

17. Eng LF, Ghirnikar RS (1994) GFAP and Astrogliosis. In: Brain Pathology. pp 229-237

18. Escartin C, Galea E, Lakatos A, O'Callaghan JP, Petzold GC, Serrano-Pozo A, Steinhäuser C, Volterra A, Carmignoto G, Agarwal A, Allen NJ, Araque A, Barbeito L, Barzilai A, Bergles DE, Bonvento G, Butt AM, Chen W-T, Cohen-Salmon M, Cunningham C, Deneen B, De Strooper B, Díaz-Castro B, Farina C, Freeman M, Gallo V, Goldman JE, Goldman SA, Götz M, Gutiérrez A, Haydon PG, Heiland DH, Hol EM, Holt MG, Iino M, Kastanenka KV, Kettenmann H, Khakh BS, Koizumi S, Lee CJ, Liddelow SA, MacVicar BA, Magistretti P, Messing A, Mishra A, Molofsky AV, Murai KK, Norris CM, Okada S, Oliet SHR, Oliveira JF, Panatier A, Parpura V, Pekna M, Pekny M, Pellerin L, Perea G, Pérez-Nievas BG, Pfrieger FW, Poskanzer KE, Quintana FJ, Ransohoff RM, Riquelme-Perez M, Robel S, Rose CR, Rothstein JD, Rouach N, Rowitch DH, Semyanov A, Sirko S, Sontheimer H, Swanson RA, Vitorica J, Wanner I-B, Wood LB, Wu J, Zheng B, Zimmer ER, Zorec R, Sofroniew MV, Verkhratsky A (2021) Reactive astrocyte nomenclature, definitions, and future directions. Nat Neurosci 24:312-325. https://doi.org/10. 1038/s41593-020-00783-4
19. Fedoroff S, McAuley WAJ, Houkle JD, Devon RM (1984) Astrocyte cell lineage. V. Similarity of astrocytes that form in the presence of dBcAMP in cultures to reactive astrocytes in vivo. J Neurosci Res 12:15-27. https://doi.org/10.1002/jnr.490120103

20. Ferroni S, Marchini C, Nobile M, Rapisarda C (1997) Characterization of an inwardly rectifying chloride conductance expressed by cultured rat cortical astrocytes. Glia 21:217-227. https:// doi.org/10.1002/(SICI)1098-1136(199710)21:2\%3c217::AIDGLIA5\%3e3.0.CO;2-3

21. Ferroni S, Marchini C, Schubert P, Rapisarda C (1995) Two distinct inwardly rectifying conductances are expressed in long term dibutyryl-cyclic-AMP treated rat cultured cortical astrocytes. FEBS Lett 367:319-325. https://doi.org/10.1016/0014-5793(95) 00588-Z

22. Ferroni S, Valente P, Caprini M, Nobile M, Schubert P, Rapisarda C (2003) Arachidonic acid activates an open rectifier potassium channel in cultured rat cortical astrocytes. J Neurosci Res 72:363-372. https://doi.org/10.1002/jnr.10580

23. Foo LC, Allen NJ, Bushong EA, Ventura PB, Chung WS, Zhou L, Cahoy JD, Daneman R, Zong H, Ellisman MH, Barres BA (2011) Development of a method for the purification and culture of rodent astrocytes. Neuron 71:799-811. https://doi.org/10.1016/j.neuron. 2011.07.022

24. Formaggio F, Saracino E, Mola MG, Rao SB, Amiry-Moghaddam M, Muccini M, Zamboni R, Nicchia GP, Caprini M, Benfenati V (2019) LRRC8A is essential for swelling-activated chloride current and for regulatory volume decrease in astrocytes. FASEB J 33:101-113. https://doi.org/10.1096/fj.201701397RR

25. Gnatenco C, Han J, Snyder AK, Kim D (2002) Functional expression of TREK-2 K+ channel in cultured rat brain astrocytes. Brain Res 931:56-67. https://doi.org/10.1016/S0006-8993(02)02261-8

26. Götz S, Bribian A, López-Mascaraque L, Götz M, Grothe B, Kunz L (2021) Heterogeneity of astrocytes: Electrophysiological properties of juxtavascular astrocytes before and after brain injury. Glia 69:346-361. https://doi.org/10.1002/glia.23900

27. Goursaud S, Kozlova EN, Maloteaux JM, Hermans E (2009) Cultured astrocytes derived from corpus callosum or cortical grey matter show distinct glutamate handling properties. J Neurochem 108:1442-1452. https://doi.org/10.1111/j.1471-4159.2009. 05889.x

28. Guatteo E, Stanness KA, Janigro D (1996) Hyperpolarizationactivated ion currents in cultured rat cortical and spinal cord astrocytes. Glia 16:196-209. https://doi.org/10.1002/(SICI)10981136(199603)16:3\%3c196::AID-GLIA2\%3e3.0.CO;2-0

29. Hibino H, Fujita A, Iwai K, Yamada M, Kurachi Y (2004) Differential assembly of inwardly rectifying $\mathrm{K}+$ channel subunits, Kir4.1 and Kir5.1, in brain astrocytes. J Biol Chem 279:4406544073. https://doi.org/10.1074/jbc.M405985200

30. Hibino H, Inanobe A, Furutani K, Murakami S, Findlay I, Kurachi Y (2010) Inwardly rectifying potassium channels: their structure, function, and physiological roles. Physiol Rev 90:291-366. https://doi.org/10.1152/physrev.00021.2009

31. Higashi K, Fujita A, Inanobe A, Tanemoto M, Doi K, Kubo T, Kurachi Y (2001) An inwardly rectifying K+ channel, Kir4.1, expressed in astrocytes surrounds synapses and blood vessels in brain. Am J Physiol - Cell Physiol 281:50-53. https://doi.org/10. 1152/ajpcell.2001.281.3.c922

32. Honsa P, Pivonkova H, Harantova L, Butenko O, Kriska J, Dzamba D, Rusnakova V, Valihrach L, Kubista M, Anderova M (2014) Increased expression of hyperpolarization-activated cyclic nucleotide-gated $(\mathrm{HCN})$ channels in reactive astrocytes following ischemia. Glia 62:2004-2021. https://doi.org/10.1002/glia.22721

33. Inyushin M, Kucheryavykh LY, Kucheryavykh YV, Nichols CG, Buono RJ, Ferraro TN, Skatchkov SN, Eaton MJ (2010) Potassium channel activity and glutamate uptake are impaired in astrocytes 
of seizure-susceptible DBA/2 mice. Epilepsia 51:1707-1713. https://doi.org/10.1111/j.1528-1167.2010.02592.x

34. Jentsch TJ, Pusch M (2018) CLC chloride channels and transporters: structure, function, physiology, and disease. Physiol Rev 98:1493-1590. https://doi.org/10.1152/physrev.00047.2017

35. Jentsch TJ, Stein V, Weinreich F, Zdebik AA (2002) Molecular structure and physiological function of chloride channels. Physiol Rev 82:503-568. https://doi.org/10.1152/physrev.00029.2001

36. Jeworutzki E, López-Hernández T, Capdevila-Nortes X, Sirisi S, Bengtsson L, Montolio M, Zifarelli G, Arnedo T, Müller CS, Schulte U, Nunes V, Martínez A, Jentsch TJ, Gasull X, Pusch M, Estévez R (2012) GlialCAM, a protein defective in a leukodystrophy, serves as a ClC-2 Cl - Channel Auxiliary Subunit. Neuron 73:951-961. https://doi.org/10.1016/j.neuron.2011.12.039

37. Jordt SE, Jentsch TJ (1997) Molecular dissection of gating in the CIC-2 chloride channel. EMBO J 16:1582-1592. https://doi.org/ 10.1093/emboj/16.7.1582

38. Kofuji P, Ceelen P, Zahs KR, Surbeck LW, Lester HA, Newman EA (2000) Genetic inactivation of an inwardly rectifying potassium channel (kir4.1 Subunit) in mice: Phenotypic impact in retina. J Neurosci 20:5733-5740. https://doi.org/10.1523/jneur osci.20-15-05733.2000

39. Kucheryavykh YV, Kucheryavykh LY, Nichols CG, Maldonado HM, Baksi K, Reichenbach A, Skatchkov SN, Eaton MJ (2007) Downregulation of Kir4.1 inward rectifying potassium channel subunits by RNAi impairs potassium transfer and glutamate uptake by cultured cortical astrocytes. Glia 55:274-281. https:// doi.org/10.1002/glia.20455

40. Lange SC, Bak LK, Waagepetersen HS, Schousboe A, Norenberg MD (2012) Primary cultures of astrocytes: their value in understanding astrocytes in health and disease. Neurochem Res 37:2569-2588. https://doi.org/10.1007/s11064-012-0868-0

41. Lee H-H, Park S-C, Choe I-S, Kim Y, Ha Y-S (2015) Time course and characteristics of astrocyte activation in the rat brain after injury. Korean J Neurotrauma 11:44. https://doi.org/10.13004/ kjnt.2015.11.2.44

42. Li L, Head V, Timpe LC (2001) Identification of an inward rectifier potassium channel gene expressed in mouse cortical astrocytes. Glia 33:57-71. https://doi.org/10.1002/1098-1136(20010 101)33:1\%3c57::AID-GLIA1006\%3e3.0.CO;2-0

43. Lim R, Mitsunobu K, Li WKP (1973) Maturation-stimulating effect of brain extract and dibutyryl cyclic AMP on dissociated embryonic brain cells in culture. Exp Cell Res 79:243-246. https://doi.org/10.1016/0014-4827(73)90512-0

44. MacFarlane SN, Sontheimer H (1997) Electrophysiological changes that accompany reactive gliosis in vitro. J Neurosci 17:7316-7329. https://doi.org/10.1523/jneurosci.17-19-07316. 1997

45. Makara JK, Petheö GL, Tóth A, Spät A (2001) pH-sensitive inwardly rectifying chloride current in cultured rat cortical astrocytes. Glia 34:52-58. https://doi.org/10.1002/glia.1039

46. Makara JK, Rappert A, Matthias K, Steinhäuser C, Spät A, Kettenmann H (2003) Astrocytes from mouse brain slices express ClC-2-mediated Cl- currents regulated during development and after injury. Mol Cell Neurosci 23:521-530. https://doi.org/10. 1016/S1044-7431(03)00080-0

47. McCarthy KD, De Vellis J (1980) Preparation of separate astroglial and oligodendroglial cell cultures from rat cerebral tissue. $\mathrm{J}$ Cell Biol 85:890-902. https://doi.org/10.1083/jcb.85.3.890

48. McKhann GM, D'Ambrosio R, Janigro D (1997) Heterogeneity of astrocyte resting membrane potentials and intercellular coupling revealed by whole-cell and gramicidin-perforated patch recordings from cultured neocortical and hippocampal slice astrocytes. J Neurosci 17:6850-6863. https://doi.org/10.1523/jneurosci.1718-06850.1997
49. McMillian MK, Thai L, Hong JS, O'Callaghan JP, Pennypacker KR (1994) Brain injury in a dish: a model for reactive gliosis. Trends Neurosci 17:138-142. https://doi.org/10.1016/01662236(94)90086-8

50. Michler-Stuke A, Wolff JR, Bottenstein JE (1984) Factors influencing astrocyte growth and development in defined media. Int $\mathbf{J}$ Dev Neurosci 2:575-584. https://doi.org/10.1016/0736-5748(84) 90035-2

51. Minieri L, Pivonkova H, Caprini M, Harantova L, Anderova M, Ferroni S (2013) The inhibitor of volume-regulated anion channels DCPIB activates TREK potassium channels in cultured astrocytes. Br J Pharmacol 168:1240-1254. https://doi.org/10.1111/ bph.12011

52. Moroni A, Barbuti A, Altomare C, Viscomi C, Morgan J, Baruscotti M, DiFrancesco D (2000) Kinetic and ionic properties of the human HCN2 pacemaker channel. Pflugers Arch Eur J Physiol 439:618-626. https://doi.org/10.1007/s004240050985

53. Moroni RF, Inverardi F, Regondi MC, Pennacchio P, Frassoni C (2015) Developmental expression of Kir4.1 in astrocytes and oligodendrocytes of rat somatosensory cortex and hippocampus. Int J Dev Neurosci 47:198-205. https://doi.org/10.1016/j.ijdevneu. 2015.09.004

54. Morrison RS, de Vellis J (1981) Growth of purified astrocytes in a chemically defined medium. Proc Natl Acad Sci U S A 78:72057209. https://doi.org/10.1073/pnas.78.11.7205

55. Neusch C, Papadopoulos N, Müller M, Maletzki I, Winter SM, Hirrlinger J, Handschuh M, Bähr M, Richter DW, Kirchhoff F, Hülsmann S (2006) Lack of the Kir4.1 channel subunit abolishes $\mathrm{K}+$ buffering properties of astrocytes in the ventral respiratory group: impact on extracellular K+ regulation. J Neurophysiol 95:1843-1852. https://doi.org/10.1152/jn.00996.2005

56. Nwaobi SE, Cuddapah VA, Patterson KC, Randolph AC, Olsen ML (2016) The role of glial-specific Kir4.1 in normal and pathological states of the CNS. Acta Neuropathol. 132

57. Nwaobi SE, Lin E, Peramsetty SR, Olsen ML (2014) DNA methylation functions as a critical regulator of Kir4.1 expression during CNS development. Glia 62:411-427. https://doi.org/10.1002/ glia. 22613

58. Olsen ML, Campbell SC, McFerrin MB, Floyd CL, Sontheimer $H$ (2010) Spinal cord injury causes a wide-spread, persistent loss of Kir4.1 and glutamate transporter 1: Benefit of 17 $\beta$-oestradiol treatment. Brain 133:1013-1025. https://doi.org/10.1093/brain/ awq049

59. Olsen ML, Campbell SL, Sontheimer H (2007) Differential distribution of Kir4.1 in spinal cord astrocytes suggests regional differences in K+ homeostasis. J Neurophysiol 98:786-793. https:// doi.org/10.1152/jn.00340.2007

60. Olsen ML, Higashimori H, Campbell SL, Hablitz JJ, Sontheimer $\mathrm{H}$ (2006) Functional expression of Kir4.1 channels in spinal cord astrocytes. Glia 53:516-528. https://doi.org/10.1002/glia.20312

61. Olsen ML, Khakh BS, Skatchkov SN, Zhou M, Lee CJ, Rouach N (2015) New insights on astrocyte ion channels: critical for homeostasis and neuron-glia signaling. J Neurosci 35:13827-13835. https://doi.org/10.1523/JNEUROSCI.2603-15.2015

62. Paco S, Hummel M, Plá V, Sumoy L, Aguado F (2016) Cyclic AMP signaling restricts activation and promotes maturation and antioxidant defenses in astrocytes. BMC Genomics 17:26232624. https://doi.org/10.1186/s12864-016-2623-4

63. Pappalardo LW, Black JA, Waxman SG Sodium Channels in Astroglia and Microglia. https://doi.org/10.1002/glia.22967

64. Päsler D, Gabriel S, Heinemann U (2007) Two-pore-domain potassium channels contribute to neuronal potassium release and glial potassium buffering in the rat hippocampus. Brain Res 1173:14-26. https://doi.org/10.1016/j.brainres.2007.07.013

65. Pekny M, Nilsson M (2005) Astrocyte activation and reactive gliosis. Glia 50:427-434. https://doi.org/10.1002/glia.20207 
66. Pekny M, Pekna M (2014) Astrocyte reactivity and reactive astrogliosis: costs and benefits. Physiol Rev 94:1077-1098. https://doi. org/10.1152/physrev.00041.2013

67. Pivonkova H, Benesova J, Butenko O, Chvatal A, Anderova M (2010) Impact of global cerebral ischemia on $\mathrm{K}+$ channel expression and membrane properties of glial cells in the rat hippocampus. Neurochem Int 57:783-794. https://doi.org/10.1016/j.neuint. 2010.08.016

68. Podda MV, Leone L, Piacentini R, Cocco S, Mezzogori D, D’Ascenzo M, Grassi C (2012) Expression of olfactory-type cyclic nucleotide-gated channels in rat cortical astrocytes. Glia 60:1391-1405. https://doi.org/10.1002/glia.22360

69. Poopalasundaram S, Knott C, Shamotienko OG, Foran PG, Dolly JO, Ghiani CA, Gallo V, Wilkin GP (2000) Glial heterogeneity in expression of the inwardly rectifying $\mathrm{K}+$ channel, Kir4.1, in adult rat CNS. Glia 30:362-372. https://doi.org/10. 1002/(SICI)1098-1136(200006)30:4\%3c362::AID-GLIA50\% 3e3.0.CO;2-4

70. Prah J, Winters A, Chaudhari K, Hersh J, Liu R, Yang SH (2019) A novel serum free primary astrocyte culture method that mimic quiescent astrocyte phenotype. J Neurosci Methods. https://doi. org/10.1016/j.jneumeth.2019.03.013

71. Ransom CB, Sontheimer H (1995) Biophysical and pharmacological characterization of inwardly rectifying $\mathrm{K}+$ currents in rat spinal cord astrocytes. J Neurophysiol 73:333-346. https://doi. org/10.1152/jn.1995.73.1.333

72. Reyes-Haro D, Miledi R, García-Colunga J (2006) Potassium currents in primary cultured astrocytes from the rat corpus callosum. J Neurocytol. https://doi.org/10.1007/s11068-006-8727-z

73. Ridet JL, Malhotra SK, Privat A, Gage FH (1997) Reactive astrocytes: cellular and molecular cues to biological function. Trends Neurosci 20:570-577. https://doi.org/10.1016/S0166-2236(97) 01139-9

74. Scholze AR, Foo LC, Mulinyawe S, Barres BA (2014) BMP Signaling in astrocytes downregulates EGFR to modulate survival and maturation. PLoS One 9https://doi.org/10.1371/journal.pone. 0110668

75. Seifert G, Hüttmann K, Binder DK, Hartmann C, Wyczynski A, Neusch C, Steinhäuser C (2009) Analysis of astroglial K+ channel expression in the developing hippocampus reveals a predominant role of the Kir4.1 subunit. J Neurosci 29:7474-7488. https://doi. org/10.1523/JNEUROSCI.3790-08.2009

76. Sen E, Basu A, Willing LB, Uliasz TF, Myrkalo JL, Vannucci SJ, Hewett SJ, Levison SW (2011) Pre-conditioning induces the precocious differentiation of neonatal astrocytes to enhance their neuroprotective properties. ASN Neuro 3:159-170. https://doi. org/10.1042/AN20100029

77. Sík A, Smith RL, Freund TF (2000) Distribution of chloride channel-2-immunoreactive neuronal and astrocytic processes in the hippocampus. Neuroscience 101:51-65. https://doi.org/10.1016/ S0306-4522(00)00360-2

78. Sirisi S, Elorza-Vidal X, Arnedo T, Armand-Ugón M, Callejo G, Capdevila-Nortes X, López-Hernández T, Schulte U, BarralloGimeno A, Nunes V, Gasull X, Estévez R (2017) Depolarization causes the formation of a ternary complex between GlialCAM, MLC1 and ClC-2 in astrocytes: implications in megalencephalic leukoencephalopathy. Hum Mol Genet 26:2436-2450. https://doi. org/10.1093/hmg/ddx134

79. Smart SL, Bosma MM, Tempel BL (1997) Identification of the delayed rectifier potassium channel, Kv1.6, in Cultured astrocytes. Glia 20:127-134. https://doi.org/10.1002/(SICI)10981136(199706)20:2\%3c127::AID-GLIA4\%3e3.0.CO;2-6
80. Tang X, Taniguchi K, Kofuji P (2009) Heterogeneity of Kir4.1 channel expression in glia revealed by mouse transgenesis. Glia 57:1706-1715. https://doi.org/10.1002/glia.20882

81. Thiemann A, Gründer S, Pusch M, Jentsch TJ (1992) A chloride channel widely expressed in epithelial and non-epithelial cells. Nature 356:57-60. https://doi.org/10.1038/356057a0

82. Verkhratsky A, Nedergaard M (2018) Physiology of astroglia. Physiol Rev 98:239-389. https://doi.org/10.1152/physrev.00042.2016

83. Verkhratsky A, Steinhäuser C (2000) Ion channels in glial cells. Brain Res Rev 32:380-412. https://doi.org/10.1016/S01650173(99)00093-4

84. Vermeiren C, De Hemptinne I, Vanhoutte N, Tilleux S, Maloteaux JM, Hermans E (2006) Loss of metabotropic glutamate receptormediated regulation of glutamate transport in chemically activated astrocytes in a rat model of amyotrophic lateral sclerosis. J Neurochem 96:719-731. https://doi.org/10.1111/j.1471-4159.2005. 03577.x

85. Vermeiren C, Najimi M, Maloteaux JM, Hermans E (2005) Molecular and functional characterisation of glutamate transporters in rat cortical astrocytes exposed to a defined combination of growth factors during in vitro differentiation. Neurochem Int 46:137-147. https://doi.org/10.1016/j.neuint.2004.08.004

86. Vermeiren C, Najimi M, Vanhoutte N, Tilleux S, De Hemptinne I, Maloteaux JM, Hermans E (2005) Acute up-regulation of glutamate uptake mediated by mGluR5a in reactive astrocytes. J Neurochem 94:405-416. https://doi.org/10.1111/j.1471-4159.2005. 03216.x

87. Walz W (2002) Chloride/anion channels in glial cell membranes. Glia 40:1-10. https://doi.org/10.1002/glia.10125

88. Wandosell F, Bovolenta P, Nieto-Sampedro M (1993) Differences between reactive astrocytes and cultured astrocytes treated with di-butyryl-cyclic AMP. J Neuropathol Exp Neurol 52:205-215. https://doi.org/10.1097/00005072-199305000-00004

89. White FP, Hertz L (1981) Protein synthesis by astrocytes in primary cultures. Neurochem Res 6:353-364. https://doi.org/10. 1007/BF00963850

90. Yarowsky PJ, Krueger BK (1989) Development of saxitoxinsensitive and insensitive sodium channels in cultured neonatal rat astrocytes. J Neurosci 9:1055-1061. https://doi.org/10.1523/ jneurosci.09-03-01055.1989

91. Zamanian JL, Xu L, Foo LC, Nouri N, Zhou L, Giffard RG, Barres BA (2012) Genomic analysis of reactive astrogliosis. J Neurosci 32:6391-6410. https://doi.org/10.1523/JNEUROSCI.6221-11.2012

92. Zhang Y, Sloan SA, Clarke LE, Caneda C, Plaza CA, Blumenthal PD, Vogel H, Steinberg GK, Edwards MSB, Li G, Duncan JA, Cheshier SH, Shuer LM, Chang EF, Grant GA, Gephart MGH, Barres BA (2016) Purification and characterization of progenitor and mature human astrocytes reveals transcriptional and functional differences with mouse. Neuron 89:37-53. https://doi.org/ 10.1016/j.neuron.2015.11.013

93. Zhou M, Kimelberg HK (2000) Freshly isolated astrocytes from rat hippocampus show two distinct current patterns and different $[\mathrm{K}+](\mathrm{O})$ uptake capabilities. J Neurophysiol 84:2746-2757. https://doi.org/10.1152/jn.2000.84.6.2746

94. Zhou M, Xu G, Xie M, Zhang X, Schools GP, Ma L, Kimelberg HK, Chen H (2009) TWIK-1 and TREK-1 are potassium channels contributing significantly to astrocyte passive conductance in rat hippocampal slices. J Neurosci 29:8551-8564. https://doi.org/10. 1523/JNEUROSCI.5784-08.2009

Publisher's note Springer Nature remains neutral with regard to jurisdictional claims in published maps and institutional affiliations. 